\title{
Parity Forwarding for Multiple-Relay Networks
}

\author{
Peyman Razaghi, Student Member, IEEE, and Wei Yu, Senior Member, IEEE
}

\begin{abstract}
This paper proposes a relaying strategy for the multiple-relay network in which each relay decodes a selection of transmitted messages by other transmitting terminals, and forwards parities of the decoded codewords. This protocol improves the previously known achievable rate of the decode-and-forward (DF) strategy for multirelay networks by allowing relays to decode only a selection of messages from relays with strong links to it. Hence, each relay may have several choices as to which messages to decode, and for a given network many different parity forwarding protocols may exist. A tree structure is devised to characterize a class of parity forwarding protocols for an arbitrary multirelay network. Based on this tree structure, closed-form expressions for the achievable rates of these DF schemes are derived. It is shown that parity forwarding is capacity achieving for new forms of degraded relay networks.
\end{abstract}

Index Terms-Achievable rate, capacity, coding, multiuser channels, relay channel, relay networks.

\section{INTRODUCTION}

A RELAY network consists of a pair of source and destination terminals and a number of relays. The relays have no message of their own and only help the source communicate to the destination. Fig. 1 shows a schematic of a network with $K$ relays in which the relays are numbered from 1 to $K$, the source is represented by index 0 , and the destination is represented by index $K+1$. The random variables $X_{0}, X_{1}, \ldots, X_{K}$ represent the transmitted signals, and $Y_{1}, Y_{2}, \ldots, Y_{K+1}$ represent the received signals, at respective nodes. The channel is assumed to be memoryless and is defined by the joint probability distribution function (pdf) $p\left(y_{1}, y_{2}, \ldots, y_{K+1} \mid x_{0}, x_{1}, \ldots, x_{K}\right)$.

Although the capacity of the simple yet fundamental singlerelay network introduced by Van der Meulen in [1] is still open, the recent surge of interests in relay networks has resulted in new communication protocols and achievable rates for multirelay networks [2]-[7]. Among the various relaying strategies, the classical decode-and-forward (DF) strategy, proposed by Cover and El Gamal [8], has been of particular interest. In the DF scheme for the single-relay channel, the relay decodes the source message and forwards a bin index for it to the destination. This fundamental relaying strategy is proved to be capacity achieving for a degraded single-relay network [8, Theorem 1].

Manuscript received November 12, 2007; revised August 24, 2008. Current version published December 24, 2008. The material in this paper has been presented in part at the IEEE International Symposium on Information Theory (ISIT), Seattle, WA, July 2006, and in part at the IEEE International Symposium on Information Theory (ISIT), Nice, France, June 2007.

The authors are with The Edward S. Rogers, Sr. Department of Electrical and Computer Engineering, University of Toronto, Toronto, ON M5S 3G4, Canada (e-mail: peyman@comm.utoronto.ca; weiyu@comm.utoronto.ca).

Communicated by P. Viswanath, Associate Editor for Communications.

Digital Object Identifier 10.1109/TIT.2008.2008131

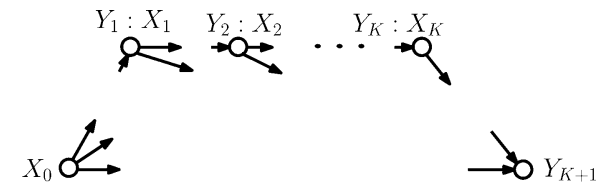

Fig. 1. A general network with multiple relays.

Generalizations of the single-relay DF scheme to the multirelay case have been studied in [2]-[6], [9]. In [3], a direct generalization of the single-relay DF strategy to the multirelay network is proposed, in which the relays successively decode messages from the previous relays and the source, then transmit bin indices of the decoded messages downstream. This successive decoding scheme is subsequently improved in [2], where a multihop scheme based on window decoding is proposed. In the multihop scheme, the source and a group of relays that have already decoded the source message replicate and cooperatively transmit the source message itself (rather than the bin indices) downstream. It has been proved that the multihop scheme is capacity achieving for a generalized multirelay version of the single-relay degraded channel [2].

This paper shows that the multihop protocol can be further improved. The main bottleneck of the multihop scheme is that all relay terminals must decode the source message. This can be restrictive, because the source rate is constrained by the decodability conditions at those relays with poor links from the source. The multihop DF rate can be improved if relays are given the flexibility of choosing an appropriate set of messages to decode. This decoding set may include not only the source message, but also messages from other relays.

This paper proposes a class of new DF strategies called parity forwarding protocol and a novel joint decoding strategy to implement the above idea. In a parity forwarding protocol, the source and the relays may transmit multiple messages. Relays decode a subset of messages transmitted by other nodes. The messages sent by relays are bin indices containing partial information for the messages in the decoding set. A key feature of the parity forwarding protocol as compared to the multihop protocol is that the relay messages partially describe the decoded data at the relays, rather than fully replicate the decoded data. Further, to decode a set of messages, the decoders (i.e., the final destination or intermediate relays) identify all messages that contain partial information about the messages to be decoded, and perform joint decoding (rather than successive decoding) by combining together all available partial information.

For a given multirelay network, many parity forwarding protocols are possible, depending on the messages decoded or transmitted by the relays. This paper characterizes a class of parity forwarding protocols in an structured way via a message tree. The message tree characterizes the dependencies 
between messages in the network. Using this tree structure, the appropriate joint decoding procedures at receiver nodes are identified and closed-form expressions for the achievable rates are derived. Further, it is shown that under certain degradedness conditions, the rates achievable by parity forwarding are the capacities.

The proposed parity forwarding protocol also includes the partial DF strategy (i.e., ([8, Theorem 7]), [10]) in which a transmitter may send multiple messages, some intended for the destination and others for downstream relays. A particular example of the partial DF strategy for the multirelay network is recently shown to be capacity achieving for a class of deterministic relay networks [11]. The parity forwarding protocol is also related to the scheme proposed in [12], [13], where a strategy of forwarding parities of each relay's observation is shown to be capacity achieving for a class of linear modulo-additive deterministic relay networks [12] and to be within a constant factor of the Gaussian relay network capacity [13].

The proposed relaying scheme is named parity forwarding, because in a linear coding context forwarding message bin indices by the relays is equivalent to forwarding parity bits. This is because parity bits of a linear code partition the linear codebook into subcodes, which are analogous to bins [14]. We use the terms "bin index" and "parity message" interchangeably throughout the paper. In a related work [15], the interpretation of bins as parities also allowed the design of practical capacity-approaching codes for the single-relay and certain forms of multirelay networks.

The rest of the paper is organized as follows. We begin with a review of existing single-relay DF schemes in Section II, and introduce joint decoding for the single-relay network. The parity forwarding protocol is introduced in Section III for a network with two relays. The general multiple-relay parity forwarding protocol is described in Section IV. Section V illustrates key features of the parity forwarding protocol through several examples. The capacity of new forms of multirelay networks are also derived in Section V. Finally, Section VI concludes the paper.

\section{Single-Relay DF: A JoInt Decoding APPROACH}

The DF scheme was first introduced in [8] for the single-relay channel and was shown to be capacity achieving for the degraded relay channel. Since then, several extensions of DF have been developed for multirelay networks ([9, Ch. 4]), [2], [3], [5], [16], [19]. In [2], the single-relay DF strategy is extended to multirelay networks and is shown to attain the capacity of the generalized multirelay version of the degraded single-relay channel. In this section, a single-relay DF approach based on joint decoding is proposed. This new approach combines the advantages of the DF methods in [8] and [2] and allows us to further improve the multirelay DF rate.

\section{A. Regular Encoding Versus Irregular Encoding}

There are several variations of the single-relay DF scheme, depending on their respective encoding and decoding methods. The two main encoding methods are the regular encoding approach of [2] (first proposed in [20] for a different channel) and the DF encoding approach originally introduced in [8], which is later named irregular encoding in [4].
A key discriminating feature of regular encoding and irregular encoding is the rate of the relay message. In regular encoding, the relay message rate is equal to the source message rate, whereas in irregular encoding, the relay message rate can be smaller than the source message rate.

In both methods, encoding is performed blockwise. Let $m_{0}^{t} \in$ $\left\{1,2, \ldots, 2^{n R_{0}}\right\}$ and $m_{1}^{t} \in\left\{1,2, \ldots, 2^{n R_{1}}\right\}$ be the source and the relay messages in block $t$. In regular encoding, the relay message in block $t$ is the source message in block $t-1$, i.e., $m_{1}^{t}=$ $m_{0}^{t-1}$, hence the relay message rate is limited to $R_{1}=R_{0}$. On the other hand, in irregular encoding, $m_{1}^{t}$ is a random bin index for $m_{0}^{t-1}$, which allows for more encoding flexibility. The bin index $m_{1}^{t}$ is computed according to $m_{1}^{t}=P_{\mathcal{B}_{1}}\left(m_{0}^{t-1}\right)$, where $P_{\mathcal{B}_{1}}(\cdot)$ is the binning function and $\mathcal{B}_{1}$ is a uniform random partition of $\left\{1,2, \ldots, 2^{n R_{0}}\right\}$ as defined in the following.

Definition 1 (Binning Function): Let $\mathcal{B}_{y}=$ $\left\{\mathcal{S}_{1}, \mathcal{S}_{2}, \ldots, \mathcal{S}_{2^{n R_{y}}}\right\}$ be a uniform random partition of $\left\{1,2, \ldots, 2^{n R_{x}}\right\}$ into $2^{n R_{y}}$ bins $\mathcal{S}_{k}$ of size $2^{n\left(R_{x}-R_{y}\right)}$ indexed by $\mathcal{Y}=\left\{1,2, \ldots, 2^{n R_{y}}\right\}$. The binning function $P_{\mathcal{B}_{y}}(\cdot)$ returns the bin index of its argument with respect to $\mathcal{B}_{y}$, i.e., $v=P_{\mathcal{B}_{y}}(u)$ if and only if $u \in \mathcal{S}_{v}$.

Codebook construction is the same for both regular and irregular encoding schemes. At the relay, $2^{n R_{1}}$ random codewords $\mathbf{x}_{1}\left(m_{1}\right)$ of length $n$ are generated according to $p\left(x_{1}\right)$ to encode $m_{1}^{t}$ in block $t$. The source codebook is constructed using superposition encoding [8] to encode both $m_{0}^{t}$ and $m_{1}^{t}$ in block $t$. This is to allow the source to cooperate with the relay, as the source in each block knows the message of the relay. More specifically, the source generates $2^{n R_{0}}$ codebooks, one for every $\mathbf{x}_{1}\left(m_{1}\right)$ codeword. For each codeword $\mathbf{x}_{1}\left(m_{1}\right), 2^{n R_{0}}$ codewords $\mathbf{x}_{1}\left(m_{0} \mid m_{1}\right)$ are randomly generated according to $p\left(x_{0} \mid x_{1}\right)$.

Both schemes give the same DF rate for the single-relay channel. However, the generalization of DF to multirelay networks is more straightforward for regular encoding, since the relay messages are replications of the source message. This results in the multihop scheme. On the other hand, irregular encoding potentially allows for DF schemes other than multihop relaying because of its flexibility with respect to the relay messages. To the best of our knowledge, multirelay DF scheme based on irregular encoding has not been proposed prior to this work, due to limitations of successive decoding when used along with irregular encoding in multirelay networks.

\section{B. Successive Decoding Versus Window Decoding}

Successive decoding [8] and window decoding [20] are the corresponding decoding approaches for the irregular and regular encoding methods, respectively (see [4] for a detailed summary of DF decoding approaches). In successive decoding, the destination first decodes the relay message, then decodes the source message with the help of the decoded relay message. The resulting constraints on the rates of the source and the relay messages to ensure successful decoding at the destination are summarized as follows [8]:

$$
\begin{aligned}
& R_{1} \leq I\left(X_{1} ; Y_{2}\right) \\
& R_{0} \leq I\left(X_{0}, X_{1} ; Y_{2}\right)
\end{aligned}
$$




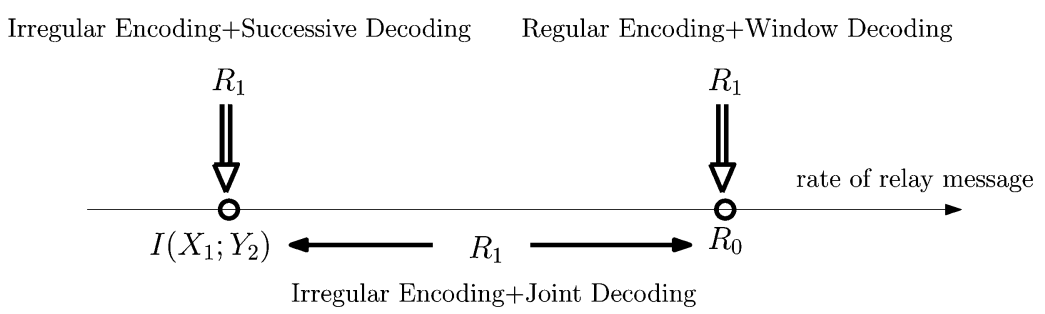

Fig. 2. If joint decoding of the source and the relay message is performed at the destination, the rate of the relay message can be flexible. This flexibility is the key to obtain higher DF rates in a multiple-relay network.

$$
R_{0} \leq I\left(X_{0} ; Y_{2} \mid X_{1}\right)+R_{1}
$$

When generalizing to multiple-relay networks, successive decoding of messages is restrictive if the relay message is to be decoded at multiple receivers (e.g., at another relay and at the final destination). In this case, because the downlink channels from the relay to different downstream receivers have different capacities, the rate of the relay message must be smaller than the minimum of the downlink capacities to ensure successful decoding of the relay message at all intended receivers (e.g., see ([3, eqs. (2)-(4)]) for constraints of this type). However, this is not optimal, since the extra rates of the downlink channels with higher capacities are wasted.

The rate limitation problem of successive decoding in multirelay networks is resolved in the multihop strategy by using regular encoding and window decoding (see [2] for the details). In regular encoding, all relays transmit replications of the source message, thus the rates of all messages transmitted over downlink channels are the same as the source rate (thus, no channel operates at a rate below its capacity). The source message is decoded by observing the received sequences over a window of successive blocks. However, the multihop protocol is not the only possible DF protocol for multiple-relay networks. The next subsection describes a joint decoding approach that allows for more flexible multirelay DF methods.

\section{Joint Decoding for Irregular Encoding}

Irregular encoding corresponds to forwarding bin indices for the received messages at the relay terminal. The key element that allows irregular encoding to be generalized to multirelay networks is a joint decoding procedure that avoids the shortcomings of successive decoding. To illustrate joint decoding, we consider the single-relay channel in this section. Joint decoding for a multirelay network follows the same principle. Note that in contrast to the multirelay networks, joint decoding has no effect on the single-relay DF rate.

The decoding procedure at the relay is similar to the one in [8] or [2] (details are omitted for brevity). The relay in each block decodes the source message provided that the source rate satisfies the following constraint:

$$
R_{0} \leq I\left(X_{0} ; Y_{1} \mid X_{1}\right)
$$

The destination jointly decodes the pair of messages $m_{0}^{t-1}$ and $m_{1}^{t}$ over the two successive blocks $t-1$ and $t$. Assume that in block $t$, the destination has already decoded $m_{0}^{t-2}$ and $m_{1}^{t-1}$ correctly. (It will become clear later that this is a valid assumption.) Knowing $m_{1}^{t-1}$ in block $t$, the destination finds a pair of messages $m_{0}$ and $m_{1}$ satisfying $m_{1}=P_{\mathcal{B}_{1}}\left(m_{0}\right)$, such that given $\mathbf{x}_{1}\left(m_{1}\right), \mathbf{x}_{0}\left(m_{0} \mid m_{1}^{t-1}\right)$ is jointly typical with $\mathbf{y}_{2}^{t-1}$, the received sequence in block $t-1$, and $\mathbf{x}_{1}\left(m_{1}\right)$ is jointly typical with $\mathbf{y}_{2}^{t}$, the received sequence in block $t$.

The probability that an incorrect $\mathbf{x}_{0}$ is jointly typical with $\mathbf{y}_{2}^{t-1}$ given $\mathbf{x}_{1}$ is asymptotically equal to $2^{-n I\left(X_{0} ; Y_{2} \mid X_{1}\right)}$ ([21, Theorem 15.2.3]); similarly, the probability that an incorrect $\mathbf{x}_{1}$ is jointly typical with $\mathbf{y}_{2}^{t}$ is asymptotically bounded by $2^{-n I\left(X_{1} ; Y_{2}\right)}$. Let $\mathcal{E}_{1}$ denote the event that $\mathbf{x}_{0}$ is decoded incorrectly, and $\mathcal{E}_{2}$ be the event that $\mathbf{x}_{1}$ is decoded incorrectly. The decoding error probability is given by $\operatorname{Pr}\left(\mathcal{E}_{1}\right)$, which can be bounded as $\operatorname{Pr}\left(\mathcal{E}_{1}\right)=\operatorname{Pr}\left(\mathcal{E}_{1} \cap\left(\mathcal{E}_{2} \cup \mathcal{E}_{2}^{c}\right)\right) \leq$ $\operatorname{Pr}\left(\mathcal{E}_{1} \cap \mathcal{E}_{2}\right)+\operatorname{Pr}\left(\mathcal{E}_{1} \cap \mathcal{E}_{2}^{c}\right)$. Now, $\operatorname{Pr}\left(\mathcal{E}_{1} \cap \mathcal{E}_{2}\right)$ is asymptotically bounded by $2^{n R_{0}} 2^{-n I\left(X_{0} ; Y_{2} \mid X_{1}\right)} 2^{-n I\left(X_{1} ; Y_{2}\right)}$. This is because $m_{1}$ is a function of $m_{0}$, hence there are $2^{n R_{0}}$ pairs of $m_{0}$ and $m_{1}$ messages in total. On the other hand, $\operatorname{Pr}\left(\mathcal{E}_{1} \cap \mathcal{E}_{2}^{c}\right)$ is asymptotically bounded by $2^{n\left(R_{0}-R_{1}\right)} 2^{-n I\left(X_{0} ; Y_{2} \mid X_{1}\right)}$, since knowing $m_{1}$ (i.e., $\mathcal{E}_{2}^{c}$ has occurred), there remain $2^{n\left(R_{0}-R_{1}\right)}$ choices for $m_{0}$. Hence, the decoding error probability at the destination asymptotically approaches zero if

$$
\begin{aligned}
R_{0} & \leq I\left(X_{0} ; Y_{2} \mid X_{1}\right)+I\left(X_{1} ; Y_{2}\right) \\
& =I\left(X_{0}, X_{1} ; Y_{2}\right) \\
R_{0} & \leq I\left(X_{0} ; Y_{2} \mid X_{1}\right)+R_{1} .
\end{aligned}
$$

Note that the rate of the relay message $R_{1}$ appears only on the right-hand side of (3) and thus is not constrained. The fact that the rate of the relay message is unconstrained is the key advantage of joint decoding as compared to successive decoding.

Joint decoding along with irregular encoding combines the benefits of irregular encoding and regular encoding by providing rate flexibility for relay messages. Fig. 2 describes the advantage of combining joint decoding and irregular encoding. In successive decoding along with irregular encoding, the relay message rate $R_{1}$ must satisfy $R_{1} \leq I\left(X_{1} ; Y_{2}\right)$. On the other hand, in window decoding along with regular encoding, the relay message has to be equal to the source message, which allows multihop type of schemes only and restricts $R_{1}$ to be equal to $R_{0}$. The combination of joint decoding and irregular encoding allows the relay messages to have any rate $R_{1}$ satisfying $I\left(X_{1} ; Y_{2}\right) \leq R_{1} \leq R_{0}$. This flexibility in choosing the rate of relay messages is the key to extend multirelay DF beyond the multihop scheme.

\section{PARITy Forwarding IN A Two-Relay Network}

We begin our discussion of the multirelay network by providing two examples of parity forwarding DF protocols for a 


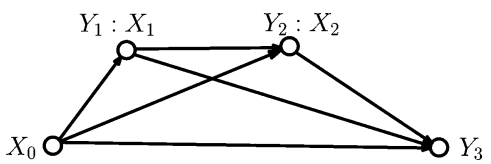

Fig. 3. A general two-relay network.

network with two relays. The first protocol, named Protocol A, demonstrates that irregular encoding along with joint decoding achieves the best previous multirelay DF rate, obtained via regular encoding in [2]. The second protocol, named Protocol B, demonstrates that the DF rate of [2] can be further improved. Protocol B also identifies the capacity of a new degraded form of two-relay networks. Later in Section V, a third two-relay DF protocol with a different achievable rate is described as a more involved example of the parity forwarding protocol. This third protocol is also capacity achieving for a class of two-relay networks under certain determinism and degradedness conditions as discussed in Section V. In general, many different DF protocols are possible in a multirelay network. Subsequent sections describe a structured characterization of a variety of DF protocols for an arbitrary multirelay network.

\section{A. Encoding}

Fig. 3 shows the schematic of a network with two relays. Let $m_{0}$ denote the source message, $m_{1}$ the message transmitted by the first relay, and $m_{2}$ the message of the second relay. In Protocol $\mathrm{A}$, the first relay decodes the source message $m_{0}$ and forwards $m_{1}$, a random bin index for $m_{0}$, to help the second relay decode $m_{0}$; the second relay decodes $m_{0}$ with the help of $m_{1}$ and forwards $m_{2}$, a random bin index for $m_{1}$; the destination decodes $m_{0}$ with the help of both $m_{1}$ and $m_{2}$. In Protocol B, again the first relay decodes the source message $m_{0}$ and forwards $m_{1}$, a bin index for $m_{0}$, to the second relay; the second relay decodes only $m_{1}$, without attempting to decode $m_{0}$, and forwards $m_{2}$, a random bin index for $m_{1}$, to the destination; the destination decodes the source message $m_{0}$ with the help of $m_{1}$ and $m_{2}$, both functions of $m_{0}$.

The difference between the two protocols is that in Protocol $\mathrm{A}$, the second relay decodes the source message $m_{0}$, whereas in Protocol B, the second relay only decodes $m_{1}$, the message of the first relay. This difference results in different achievable DF rates for the two protocols. This can be best observed if we consider the following extreme cases: if the channel from the source to the second relay is completely blocked and the source can only communicate to the second relay through the first relay, Protocol A achieves a lower rate as compared to Protocol B, since in Protocol A the source data rate is bounded by the decodability condition of the source message at the relay, whereas the rate of the source message in Protocol B is not so constrained. However, if the channel from the source to the second relay is strong, then Protocol A may give a higher rate, since in Protocol A, the second relay can take advantage of $m_{1}$ when decoding $m_{0}$, whereas in Protocol B, the second relay receives no extra help when decoding $m_{1}$.

The encoding scheme is the same for both protocols. A binning scheme along with block Markov encoding is performed. Let $n$ denote the length of each transmission block. In block $t$, the messages of the source, the first relay, and the second relay, $m_{0}^{t}, m_{1}^{t}$, and $m_{2}^{t}$ are selected from the sets $\left\{1,2, \ldots, 2^{n R_{0}}\right\}$, $\left\{1,2, \ldots, 2^{n R_{1}}\right\}$, and $\left\{1,2, \ldots, 2^{n R_{2}}\right\}$, respectively. In each block, the message of the first relay is a random bin index (parity message) for the previous source message, and the message of the second relay is a parity message for the previous message of the first relay. The three messages in the network are related as $m_{1}^{t}=P_{\mathcal{B}_{1}}\left(m_{0}^{t-1}\right)$, and $m_{2}^{t}=P_{\mathcal{B}_{2}}\left(m_{1}^{t-1}\right)$, where $\mathcal{B}_{1}$ and $\mathcal{B}_{2}$ are random partitions of sizes $2^{n R_{1}}$ and $2^{n R_{2}}$, respectively.

Random codebooks are used to encode the three messages. To encode $m_{2}$ at the second relay, $2^{n R_{2}}$ random codewords $\mathbf{x}_{2}\left(m_{2}\right)$ of length $n$ are generated according to $p\left(x_{2}\right)$. The codebook used at the first relay depends on the codeword transmitted by the second relay. This is to allow the first relay to cooperate with the second relay. As the first relay in each block knows the message of the second relay, it can generate $2^{n R_{2}}$ codebooks, one for each one of $\mathbf{x}_{2}\left(m_{2}\right)$ codewords. To generate a codebook depending on the codeword $\mathbf{x}_{2}\left(m_{2}\right), 2^{n R_{1}}$ codewords $\mathbf{x}_{1}\left(m_{1} \mid m_{2}\right)$ are randomly generated according to $p\left(x_{1} \mid x_{2}\right)$. Similarly, in order to allow the source to cooperate with the messages of the first and the second relay, which are known to the source in each block, the source codebook is constructed by generating $2^{n R_{0}}$ codewords $\mathbf{x}_{0}\left(m_{0} \mid m_{1}, m_{2}\right)$ for each pair of $m_{1}$ and $m_{2}$ messages, according to $p\left(x_{0} \mid x_{1}, x_{2}\right)$. This is an instance of superposition broadcast or briefly superposition encoding, as it is similar to the superposition codebook construction for the degraded broadcast channel ([21, Ch. 14]).

\section{B. Decoding}

Decoding at the relay and at the destination is performed using joint typicality test. In block $t$, the first relay knows $m_{1}^{t}$ and $m_{2}^{t}=P_{\mathcal{B}_{2}}\left(m_{1}^{t-1}\right)$. To decode $m_{0}^{t}$, the first relay finds a codeword $\mathbf{x}_{0}\left(m_{0} \mid m_{1}^{t}, m_{2}^{t}\right)$ that is jointly typical with its received sequence $\mathbf{y}_{1}^{t}$ given $\mathbf{x}_{2}\left(m_{2}^{t}\right)$ and $\mathbf{x}_{1}\left(m_{1}^{t} \mid m_{2}^{t}\right)$. The probability that given $\mathbf{x}_{2}\left(m_{2}^{t}\right)$ and $\mathbf{x}_{1}\left(m_{1}^{t} \mid m_{2}^{t}\right)$, an incorrect codeword $\mathbf{x}_{0}$, independent of $\mathbf{y}_{1}^{t}$, is jointly typical with $\mathbf{y}_{1}^{t}$ is asymptotically bounded by $2^{-n I\left(X_{0} ; Y_{1} \mid X_{1}, X_{2}\right)}$ ([21, Theorem 15.2.3]). Since there are $2^{n R_{0}}$ possibilities for $m_{0}$, the decoding at the first relay is successful asymptotically with zero error probability if

$$
R_{0} \leq I\left(X_{0} ; Y_{1} \mid X_{1}, X_{2}\right) .
$$

The required constraint to ensure asymptotical zero probability of the decoding error at the second relay is different for the two protocols. In the following, each protocol is considered separately.

1) Rate Constraints for the Second Relay in Protocol A: The second relay in Protocol A decodes the source message with the help of the message of the first relay. More specifically, in block $t$, the second relay decodes $m_{0}^{t-1}$ with the help of $m_{1}^{t}=$ $P_{\mathcal{B}_{1}}\left(m_{0}^{t-1}\right)$. Assume that $m_{0}^{t-2}$ is successfully decoded prior to block $t$. Knowing $m_{2}^{t}$ and $m_{1}^{t-1}$ in block $t$, the second relay decodes the source message over two successive blocks by finding a pair of messages $m_{0}$ and $m_{1}$ satisfying $m_{1}=P_{\mathcal{B}_{1}}\left(m_{0}\right)$, such that $\mathbf{x}_{0}\left(m_{0} \mid m_{1}, m_{2}^{t-1}\right)$ is jointly typical with $\mathbf{y}_{2}^{t-1}$ given $\mathbf{x}_{1}\left(m_{1} \mid m_{2}^{t-1}\right)$ and $\mathbf{x}_{2}\left(m_{2}^{t-1}\right)$, and $\mathbf{x}_{1}\left(m_{1} \mid m_{2}^{t}\right)$ is jointly typical with $\mathbf{y}_{2}^{t}$ given $\mathbf{x}_{2}\left(m_{2}^{t}\right)$. 
The probability that an incorrect $\mathbf{x}_{0}$ is jointly typical with $\mathbf{y}_{2}^{t-1}$ given $\mathbf{x}_{1}$ and $\mathbf{x}_{2}$, is asymptotically bounded by $2^{-n I\left(X_{0} ; Y_{2} \mid X_{1}, X_{2}\right)}$; the probability that an incorrect $\mathbf{x}_{1}$ is jointly typical with $\mathbf{y}_{2}^{t}$ given $\mathbf{x}_{2}$ is asymptotically bounded by $2^{-n I\left(X_{1} ; Y_{2} \mid X_{2}\right)}$. The analysis of the probability of error closely follows the one in Section II-C. Let $\mathcal{E}_{1}$ denote the event that $\mathbf{x}_{0}$ is decoded incorrectly, and $\mathcal{E}_{2}$ be the event that $\mathbf{x}_{1}$ is decoded incorrectly. The decoding error probability is given by $p\left(\mathcal{E}_{1}\right)$, which can be bounded as $\operatorname{Pr}\left(\mathcal{E}_{1}\right)=\operatorname{Pr}\left(\mathcal{E}_{1} \cap\left(\mathcal{E}_{2} \cup \mathcal{E}_{2}^{c}\right)\right) \leq$ $\operatorname{Pr}\left(\mathcal{E}_{1} \cap \mathcal{E}_{2}\right)+\operatorname{Pr}\left(\mathcal{E}_{1} \cap \mathcal{E}_{2}^{c}\right)$. Now, $\operatorname{Pr}\left(\mathcal{E}_{1} \cap \mathcal{E}_{2}\right)$ is asymptotically bounded by $2^{n R_{0}} 2^{-n I\left(X_{0} ; Y_{2} \mid X_{1}, X_{2}\right)} 2^{-n I\left(X_{1} ; Y_{2} \mid X_{2}\right)}$ as there are $2^{n R_{0}}$ pairs of $m_{0}$ and $m_{1}$ messages in total (since $m_{1}$ is a function of $\left.m_{0}\right)$. Similarly, $\operatorname{Pr}\left(\mathcal{E}_{1} \cap \mathcal{E}_{2}^{c}\right)$ is asymptotically bounded by $2^{n\left(R_{0}-R_{1}\right)} 2^{-n I\left(X_{0} ; Y_{2} \mid X_{1}, X_{2}\right)}$, since knowing $m_{1}$, there remain $2^{n\left(R_{0}-R_{1}\right)}$ choices for $m_{0}$. Hence, the decoding error probability at the second relay is asymptotically zero if

$$
\begin{aligned}
R_{0} & \leq I\left(X_{0} ; Y_{2} \mid X_{1}, X_{2}\right)+I\left(X_{1} ; Y_{2} \mid X_{2}\right) \\
& =I\left(X_{0}, X_{1} ; Y_{2} \mid X_{2}\right) \\
R_{0} & \leq I\left(X_{0} ; Y_{2} \mid X_{1}, X_{2}\right)+R_{1} .
\end{aligned}
$$

2) Rate Constraints for the Second Relay in Protocol B: In Protocol B, the second relay only decodes the message of the first relay, i.e., in block $t$, the second relay decodes $m_{1}^{t}$. Decoding is performed by finding a codeword $\mathbf{x}_{1}\left(m_{1}\right)$ that is jointly typical with $\mathbf{y}_{2}^{t}$ given $\mathbf{x}_{2}\left(m_{2}^{t}\right)$. The probability that a codeword $\mathbf{x}_{1}$, independent of $\mathbf{y}_{2}^{t}$, is incorrectly decoded as the transmitted codeword by the first relay is asymptotically bounded by $2^{-n I\left(X_{1} ; Y_{2} \mid X_{2}\right)}$. Hence, successful decoding at the second relay is possible asymptotically if

$$
R_{1} \leq I\left(X_{1} ; Y_{2} \mid X_{2}\right) .
$$

3) Rate Constraints at the Destination: The required rate constraints at the destination to ensure asymptotically zero probability of decoding error are the same for both protocols. The decoding procedure at the destination is similar to the one at the second relay in Protocol A. The destination decodes the source message with the help of the messages of the first relay and the second relay. Specifically, in block $t$, the destination decodes $m_{0}^{t-2}$ with the help of $m_{1}^{t-1}=P_{\mathcal{B}_{1}}\left(m_{0}^{t-2}\right)$ and $m_{2}^{t}=P_{\mathcal{B}_{2}}\left(m_{1}^{t-1}\right)$. Decoding is performed by jointly finding three messages $m_{0}, m_{1}$, and $m_{2}$, such that the codeword $\mathbf{x}_{2}\left(m_{2}\right)$ is jointly typical with $\mathbf{y}_{3}^{t} ; \mathbf{x}_{1}\left(m_{1} \mid m_{2}\right)$ is jointly typical with $\mathbf{y}_{3}^{t-1}$ given $\mathbf{x}_{2}\left(m_{2}\right)$; and $\mathbf{x}_{0}\left(m_{0} \mid m_{1}, m_{2}\right)$ is jointly typical with $\mathbf{y}_{3}^{t-2}$ given $\mathbf{x}_{1}\left(m_{1} \mid m_{2}\right)$ and $\mathbf{x}_{2}\left(m_{2}\right)$. Asymptotically for large $n$, the error probability of the joint typicality test of $\mathbf{x}_{2}$ and $\mathbf{y}_{3}^{t}$ is equal to $2^{-n I\left(X_{2} ; Y_{3}\right)}$; the error probability of the joint typicality test of $\mathbf{x}_{1}$ and $\mathbf{y}_{3}^{t-1}$ given $\mathbf{x}_{2}$, is $2^{-n I\left(X_{1} ; Y_{3} \mid X_{2}\right)}$; and the error probability of the joint typicality test of $\mathbf{x}_{0}$ and $\mathbf{y}_{3}^{t-2}$ given $\mathbf{x}_{1}$ and $\mathbf{x}_{2}$, is $2^{-n I\left(X_{0} ; Y_{3} \mid X_{1}, X_{2}\right)}$. There are $2^{n R_{0}}$ valid combinations ${ }^{1}$ of $m_{0}, m_{1}$, and $m_{2}$ messages; $2^{n\left(R_{0}-R_{2}\right)}$ combinations of $m_{0}$ and $m_{1}$ messages for a given $m_{2}$; and $2^{n\left(R_{0}-R_{1}\right)}$ choices for $m_{0}$ given the two fixed bin indices $m_{1}$ and $m_{2}$ (note that $m_{2}$ is also fixed when $m_{1}$ is fixed). Consequently, by an analysis similar to the one for (5), the

\footnotetext{
${ }^{1} \mathrm{~A}$ valid combination corresponds to a set of values for $m_{0}, m_{1}$, and $m_{2}$ such that $m_{2}=P_{\mathcal{B}_{2}}\left(m_{1}\right)$ and $m_{1}=P_{\mathcal{B}_{1}}\left(m_{0}\right)$.
}

probability of decoding error at the destination approaches zero asymptotically if the following constraints are satisfied:

$$
\begin{aligned}
R_{0} \leq & I\left(X_{0} ; Y_{3} \mid X_{1}, X_{2}\right)+I\left(X_{1} ; Y_{3} \mid X_{2}\right) \\
& +I\left(X_{2} ; Y_{3}\right) \\
= & I\left(X_{0}, X_{1}, X_{2} ; Y_{3}\right) \\
R_{0} \leq & I\left(X_{0} ; Y_{3} \mid X_{1}, X_{2}\right)+I\left(X_{1} ; Y_{3} \mid X_{2}\right)+R_{2} \\
R_{0} \leq & I\left(X_{0} ; Y_{3} \mid X_{1}, X_{2}\right)+R_{1} .
\end{aligned}
$$

The following theorems summarize the achievable rates of the two protocols for a two-relay network.

Theorem 1 (Achievable Rate of Protocol A): For a memoryless two-relay network defined by $p\left(y_{1}, y_{2}, y_{3} \mid x_{0}, x_{1}, x_{2}\right)$, fixing any $p\left(x_{0}, x_{1}, x_{2}\right)$, the source rate $R_{0}$ satisfying the following constraints is achievable:

$$
\begin{aligned}
& R_{0} \leq I\left(X_{0} ; Y_{1} \mid X_{1}, X_{2}\right) \\
& R_{0} \leq I\left(X_{0}, X_{1} ; Y_{2} \mid X_{2}\right) \\
& R_{0} \leq I\left(X_{0}, X_{1}, X_{2} ; Y_{3}\right) .
\end{aligned}
$$

Proof: The above rate is obtained by combining (4), (5), and (7), and using the fact that constraints involving $R_{1}$ and $R_{2}$ can be ignored, since $R_{1}$ and $R_{2}$ only appear on the right-hand side of all the inequalities and can be increased freely. This rate is previously derived in [2] using a regular encoding approach. $\square$

Theorem 2 (Achievable Rate of Protocol B): For a memoryless two-relay network defined by $p\left(y_{1}, y_{2}, y_{3} \mid x_{0}, x_{1}, x_{2}\right)$, fixing any $p\left(x_{0}, x_{1}, x_{2}\right)$, the source rate $R_{0}$ satisfying the following constraints is achievable:

$$
\begin{aligned}
& R_{0} \leq I\left(X_{0} ; Y_{1} \mid X_{1}, X_{2}\right) \\
& R_{0} \leq I\left(X_{0} ; Y_{3} \mid X_{1}, X_{2}\right)+I\left(X_{1} ; Y_{2} \mid X_{2}\right) \\
& R_{0} \leq I\left(X_{0}, X_{1}, X_{2} ; Y_{3}\right) .
\end{aligned}
$$

Proof: The above rate constraints are derived from (4), (6), (7). Note that (9b) is a consequence of (7c) and (6). Also, (7b) can be ignored, since $R_{2}$ appears only on the right-hand side and can be freely increased.

\section{Protocol A Versus Protocol B}

The rate achieved by Protocol A can be higher or lower than the rate achieved by Protocol B depending on channel parameters. Protocol A can be capacity achieving if the network is degraded in a particular way. Protocol B can also be capacity achieving, however, under a different degradedness condition.

The cut-set bound can be used to identify networks for which Protocols A and B are capacity achieving. According to the cut-set bound for a two-relay network, the source rate $R_{0}$ satisfies the following inequalities for some joint distribution $p\left(x_{0}, x_{1}, x_{2}\right)([21$, Ch. 14] $):$

$$
\begin{aligned}
& R_{0} \leq I\left(X_{0} ; Y_{1}, Y_{2}, Y_{3} \mid X_{1}, X_{2}\right) \\
& R_{0} \leq I\left(X_{0}, X_{1} ; Y_{2}, Y_{3} \mid X_{2}\right) \\
& R_{0} \leq I\left(X_{0}, X_{1}, X_{2} ; Y_{3}\right) .
\end{aligned}
$$

Protocol A is capacity achieving for a two-relay network in which $X_{0}-\left(X_{1}, X_{2}, Y_{1}\right)-\left(Y_{2}, Y_{3}\right)$ and $\left(X_{0}, X_{1}\right)$ - 
$\left(X_{2}, Y_{2}\right)-Y_{3}$ form Markov chains [2]. For such a network, the rate (9) is equivalent to (10), since $I\left(X_{0} ; Y_{1}, Y_{2}, Y_{3} \mid X_{1}, X_{2}\right)=$ $I\left(X_{0} ; Y_{1} \mid X_{1}, X_{2}\right)$ if $X_{0}-\left(X_{1}, X_{2}, Y_{1}\right)-\left(Y_{2}, Y_{3}\right)$ is a Markov chain, and $I\left(X_{0}, X_{1} ; Y_{2}, Y_{3} \mid X_{2}\right)=I\left(X_{0}, X_{1} ; Y_{2} \mid X_{2}\right)$ if $\left(X_{0}, X_{1}\right)-\left(X_{2}, Y_{2}\right)-Y_{3}$ is a Markov chain.

On the other hand, Protocol B achieves the capacity of a tworelay network in which $X_{0}-\left(X_{1}, X_{2}, Y_{1}\right)-\left(Y_{2}, Y_{3}\right), X_{1}-$ $\left(X_{2}, Y_{2}\right)-Y_{3}$, and $X_{0}-\left(X_{1}, X_{2}, Y_{3}\right)-Y_{2}$ form Markov chains. For this channel, the equivalence of (10a) and (9a) is a direct consequence of $X_{0}-\left(X_{1}, X_{2}, Y_{1}\right)-\left(Y_{2}, Y_{3}\right)$. It remains to show that (10b) reduces to (9b), which can be proved as follows:

$$
\begin{aligned}
& I\left(X_{0}, X_{1} ; Y_{2}, Y_{3} \mid X_{2}\right) \\
& \stackrel{(a)}{=} I\left(X_{1} ; Y_{2}, Y_{3} \mid X_{2}\right)+I\left(X_{0} ; Y_{2}, Y_{3} \mid X_{1}, X_{2}\right) \\
& \stackrel{(b)}{=} I\left(X_{1} ; Y_{2} \mid X_{2}\right)+I\left(X_{0} ; Y_{2}, Y_{3} \mid X_{1}, X_{2}\right) \\
& \stackrel{(c)}{=} I\left(X_{1} ; Y_{2} \mid X_{2}\right)+I\left(X_{0} ; Y_{3} \mid X_{1}, X_{2}\right)
\end{aligned}
$$

where (a) follows from the chain rule for the mutual information, (b) follows from $X_{1}-\left(X_{2}, Y_{2}\right)-Y_{3}$, and (c) follows from $X_{0}-\left(X_{1}, X_{2}, Y_{3}\right)-Y_{2}$. For future reference, we call this type of degraded network doubly degraded.

Doubly degraded network corresponds to a network in which the channel from the source to the second relay is blocked. The interpretation of the first Markov chain $X_{0}-\left(X_{1}, X_{2}, Y_{1}\right)$ $\left(Y_{2}, Y_{3}\right)$ is that the channel from the source to the first relay is stronger than the channel from the source to the second relay and the destination. The second Markov chain $X_{1}-\left(X_{2}, Y_{2}\right)-Y_{3}$ states that the channel from the first relay to the second relay is stronger than the channel from the first relay to the destination. The last Markov chain $X_{0}-\left(X_{1}, X_{2}, Y_{3}\right)-Y_{2}$ implies that the channel from the source to the second relay is weaker than the channel from the source to the destination. The next example describes a Gaussian version of such a doubly degraded network.

Example: Consider the two-relay network depicted in Fig. 4. In this network, the source signal is represented by $X_{0}$. The first relay receives $Y_{1}=X_{0}+N_{1}$ where $N_{1} \sim \mathcal{N}\left(0, \sigma_{1}^{2}\right)$ and transmits $X_{1}$. The channel from the source to the second relay is blocked. The second relay receives $Y_{2}=X_{1}+N_{2}$, and transmits

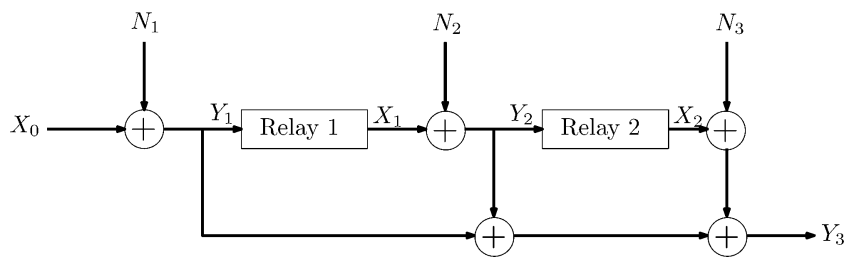

Fig. 4. A Gaussian doubly degraded two-relay network.

$X_{2}$, where $N_{2} \sim \mathcal{N}\left(0, \sigma_{2}^{2}\right)$. Destination receives $Y_{3}=X_{0}+$ $X_{1}+X_{2}+N_{1}+N_{2}+N_{3}$, where $N_{3} \sim \mathcal{N}\left(0, \sigma_{3}^{2}\right)$.

It can be shown that a joint Gaussian distribution is the optimal input distribution for such a degraded additive Gaussian noise network. This is proved for the degraded single-relay channel with additive Gaussian noise in [8, Sec. IV]. The same technique is applicable to the multirelay case. We skip the proof and assume the optimality of the jointly Gaussian input in the following.

The achievable rate of Protocol B is derived by defining the joint distribution of $\left(X_{0}, X_{1}, X_{2}\right)$ as follows. Let $X_{0}=X_{0}^{\prime}+$ $\alpha_{11} X_{1}^{\prime}+\alpha_{12} X_{2}^{\prime}$ where $X_{0}^{\prime} \sim \mathcal{N}\left(0, Q_{0}\right), X_{1}^{\prime} \sim \mathcal{N}\left(0, Q_{1}\right)$, and $X_{2}^{\prime} \sim \mathcal{N}\left(0, Q_{2}\right)$ are independent Gaussian random variables. For the first relay, let $X_{1}=X_{1}^{\prime}+\alpha_{22} X_{2}^{\prime}$, and for the second relay, set $X_{2}=X_{2}^{\prime}$. The correlations among the three input signals are controlled by $\alpha_{i j}, 1 \leq i \leq j \leq 2$.

The network is doubly degraded, since the source can only communicate to the destination through the first relay, the first relay can only communicate to the destination through the second relay, and the source cannot directly communicate to the second relay.

The achievable rate of Protocol A under power constraints $\mathbb{E} X_{i}^{2} \leq P_{i}, 0 \leq i \leq 2$ is given by (11a)-(11c) shown at the bottom of the page, maximized over $\alpha_{i j}, 1 \leq i \leq j \leq 2$, $Q_{0}, Q_{1}$, and $Q_{2}$ such that $Q_{0}+\alpha_{11}^{2} Q_{1}+\alpha_{12}^{2} Q_{2} \leq P_{0}, Q_{1}+$ $\alpha_{22}^{2} Q_{2} \leq P_{1}$, and $Q_{2} \leq P_{2}$. This rate is also equal to the multihop rate of [2] for this network.

The power-constrained achievable rate of Protocol B given in (9) translates into the constraints, shown in (12a)-(12c) also at the bottom of the page, maximized over $\alpha_{i j}, 1 \leq i \leq j \leq 2$, $Q_{0}, Q_{1}$, and $Q_{2}$ such that $Q_{0}+\alpha_{11}^{2} Q_{1}+\alpha_{12}^{2} Q_{2} \leq P_{0}, Q_{1}+$

$$
\begin{aligned}
R_{0} & <\frac{1}{2} \log _{2}\left(1+\frac{Q_{0}}{\sigma_{1}^{2}}\right) \\
R_{0} & <\frac{1}{2} \log _{2}\left(1+\frac{Q_{1}}{\sigma_{2}^{2}}\right) \\
R_{0} & <\frac{1}{2} \log _{2}\left(1+\frac{Q_{0}+\left(1+\alpha_{11}\right)^{2} Q_{1}+\left(1+\alpha_{12}+\alpha_{22}\right)^{2} Q_{2}}{\sigma_{1}^{2}+\sigma_{2}^{2}+\sigma_{3}^{2}}\right) .
\end{aligned}
$$

$$
\begin{aligned}
R_{0} & <\frac{1}{2} \log _{2}\left(1+\frac{Q_{0}}{\sigma_{1}^{2}}\right) \\
R_{0} & <\frac{1}{2} \log _{2}\left(1+\frac{Q_{0}}{\sigma_{1}^{2}+\sigma_{2}^{2}+\sigma_{3}^{2}}\right)+\frac{1}{2} \log _{2}\left(1+\frac{Q_{1}}{\sigma_{2}^{2}}\right) \\
R_{0} & <\frac{1}{2} \log _{2}\left(1+\frac{Q_{0}+\left(1+\alpha_{11}\right)^{2} Q_{1}+\left(1+\alpha_{12}+\alpha_{22}\right)^{2} Q_{2}}{\sigma_{1}^{2}+\sigma_{2}^{2}+\sigma_{3}^{2}}\right)
\end{aligned}
$$




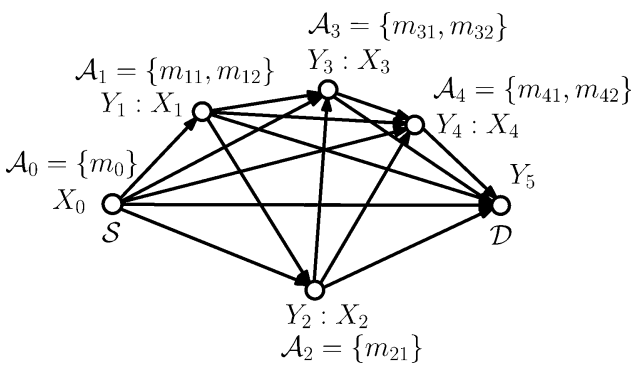

Fig. 5. A network with four relays. The message sets $\mathcal{A}_{0}$ up to $\mathcal{A}_{4}$ specify the messages each relay encodes.

$\alpha_{22}^{2} Q_{2} \leq P_{1}$, and $Q_{2} \leq P_{2}$. This rate, which is also the capacity of this doubly degraded network, is strictly greater than the multihop rate (11). As a comparison of Protocols A and B, note that in the case that the optimal values of $Q_{0}, Q_{1}, \sigma_{1}^{2}$, and $\sigma_{2}^{2}$ are such that $Q_{1} / \sigma_{2}^{2}<Q_{0} / \sigma_{1}^{2}$ (e.g., $\sigma_{1}^{2} \ll \sigma_{2}^{2}$ ), it is better to turn the second relay off rather than forcing it to decode the source message in Protocol A; however, the second relay can still help the overall transmission using Protocol B.

\section{MultiRElay PARITY ForWARDING}

The parity forwarding protocol can be generalized to multirelay networks by allowing relay terminals to transmit messages that are bin indices of the messages of the source or other relays. The first step in such a structured generalization is to specify the relation among messages.

The relation among messages in a parity forwarding protocol is transitive and can take various forms. For example, a relay message $\mathrm{A}$ can be a random bin index of another relay message $B$, while message $B$ itself is a bin index of another message C. Thus, message $\mathrm{C}$ is also a bin index for message $\mathrm{A}$. Another possibility could be that messages $\mathrm{B}$ and $\mathrm{C}$ are independent random bin indices of message A. In this paper, we propose the use of a message tree to describe the relation among messages.

Consider an example of a parity forwarding protocol in a network with four relays shown in Fig. 5. Fig. 6 shows a message tree that specifies one possible relationship between messages and their parities. In the message tree, a node represents a message. The root node represents the source message $m_{0}$. Branching out from a message corresponds to forming a parity for that message, i.e., over each branch, the child message is a parity (random bin index) for the parent message.

There are several possibilities for associating messages in the tree with transmitting nodes. The message sets $\mathcal{A}_{0}, \mathcal{A}_{1}, \mathcal{A}_{2}, \mathcal{A}_{3}, \mathcal{A}_{4}$ in Fig. 5 describe one such association for the message tree in Fig. 6. In general, the message sets partition the set of all messages in the tree and each partition is associated with a transmitter (source or relay). Several partition schemes may exist for a message tree, resulting in different parity forwarding protocols with different rates.

A relay node computes the messages associated with it by decoding a set of messages from other nodes. For example, all parity messages are computable if the relay decodes the source message $m_{0}$. However, decoding the source message is not the only possibility. For example, the second relay in Fig. 6 only needs to decode the message $m_{11}$ in order to compute $\mathcal{A}_{2}=$

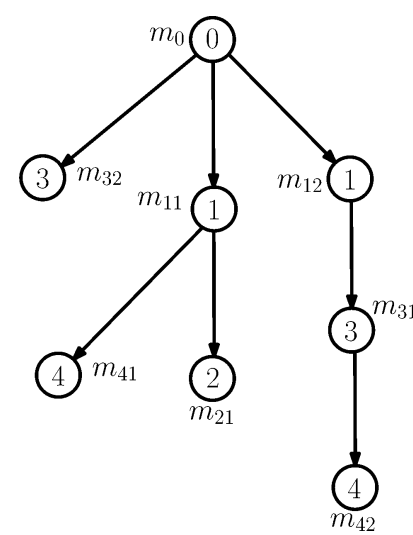

Fig. 6. An example of message tree and message sets for a network with four relays shown in Fig. 5.

$\left\{m_{21}\right\}$. The set of messages that a relay decodes is called the decoding message set. For the second relay in this example, the decoding set can be $\mathcal{D}_{2}=\left\{m_{0}\right\}$, or $\mathcal{D}_{2}=\left\{m_{11}\right\}$, or $\mathcal{D}_{2}=\left\{m_{11}, m_{12}\right\}$, because the message set of the second relay $\mathcal{A}_{2}=\left\{m_{21}\right\}$ is directly a parity of $m_{11}$ and indirectly a parity of $m_{0}$. For the destination, the decoding set is always the set $\left\{m_{0}\right\}$. Different choices of the decoding sets result in different rates corresponding to different parity forwarding protocols. For example, for the network with two relays in the previous section, different DF rates are achieved depending on which message the second relay decodes.

In summary, a parity forwarding protocol for a multirelay network is characterized by three components:

- a message tree which specifies a set of messages and their relation with respect to each other;

- the message sets which associate messages in the message tree with the transmitters (source or the relays); and

- the decoding sets which specify the messages that the relays decode.

\section{A. Mathematical Formulation}

Formally, consider a general multirelay network consisting of a pair of source and destination and $K$ relays numbered from 1 to $K$. Let the set $\mathcal{A}_{0}=\left\{m_{01}, m_{02}, \ldots, m_{0 n_{0}}\right\}$ denote the set of messages encoded by the source, where $m_{0 n_{0}}$ is the source message and the rest of messages are parities for $m_{0 n_{0}}$. For notational simplicity, let the subscript " $0 n_{0}$ " be equivalent to " 0 " so that, for example, $m_{0}=m_{0 n_{0}}$. In each block, the $k$ th relay, $1 \leq k \leq K$, transmits a set of $n_{k}>0$ parity messages $\mathcal{A}_{k}=\left\{m_{k 1}, m_{k 2}, \ldots, m_{k n_{k}}\right\}$. The message tree, defined in the following, specifies the relation among the messages.

Definition 2 (Message Tree): The message tree is defined by a directed tree $\tau=(\mathcal{M}, \mathcal{V})$ where $\mathcal{M}$ denotes the set of nodes and $\mathcal{V}$ denotes the set of directed edges. Each node in the message tree represents a message. The source message $m_{0}$ is associated with the root node. All other nodes correspond to parity messages sent by the relay terminals. An edge corresponds to forming a bin index. Branching out from the message $m_{k^{\prime} l^{\prime}}$ to the message $m_{k l}$ corresponds to forming the bin index $m_{k l}$ for the message $m_{k^{\prime} l^{\prime}}$ with respect to a random partition $\mathcal{B}_{k l}$ of the message space $\left\{1,2, \ldots, 2^{n R_{k^{\prime} l^{\prime}}}\right\}$ into $2^{n R_{k l}}$ 
bins, i.e., $m_{k l}=P_{\mathcal{B}_{k l}}\left(m_{k^{\prime}} l^{\prime}\right)$. The random partition sets $\mathcal{B}_{k l}$, $1 \leq k \leq K, 1 \leq l \leq n_{k}$ are generated independently.

Notation $1(\rightarrow, \nrightarrow)$ : We use $m \rightarrow m^{\prime}$ to denote that the message $m^{\prime}$ is a descendent of the message $m$. The message $m^{\prime}$ is a descendent of $m$ if there is a path from the message $m$ to the message $m^{\prime}$ in the message tree, i.e., $m^{\prime}$ is a parity message for $m$, either directly or indirectly via other intermediate parity messages. A message is considered to be a descendent (parity) of itself, i.e., $\forall m \in \mathcal{M}, m \rightarrow m$. We write $m \nrightarrow m^{\prime}$ if $m \rightarrow$ $m^{\prime}$ is not true. We write $\mathcal{F} \rightarrow m$ to denote that the message $m$ is a descendent of the set $\mathcal{F} \subset \mathcal{M}$. The message $m$ is a descendent of $\mathcal{F}$ if $\exists f \in \mathcal{F}$ such that $f \rightarrow m$. We write $\mathcal{F} \rightarrow \mathcal{G}$ to denote that the set $\mathcal{G} \subset \mathcal{M}$ is a descendent of $\mathcal{F}$. The set $\mathcal{G}$ is a descendent of $\mathcal{F}$ if $\mathcal{F} \rightarrow g, \forall g \in \mathcal{G}$; in this case, $\mathcal{F}$ is called a generator for $\mathcal{G}$. The " $\nrightarrow$ " relation for sets of messages denotes the negation of " $\rightarrow$ ".

The collection of the message sets $\mathcal{A}_{k}$ 's forms a disjoint partition of $\mathcal{M}$. The $\mathcal{A}_{k}$ sets are such that for $i<j$, there are no messages in $\mathcal{A}_{i}$ that are a descendent of $\mathcal{A}_{j}$. This is required since messages in $\mathcal{A}_{k}$ are constructed by forming parities for messages in $\mathcal{A}_{0}, \mathcal{A}_{1}, \ldots, \mathcal{A}_{k-1}$. A message is said to be of order $k$ if it is transmitted by the $k$ th relay. Note that a message and its parity may be transmitted by the same relay; this allows for partial decode-and-forward schemes of the type introduced in ([8, Theorem 7] (see the example in Section V-E).

Having specified the relation of messages with each other, we now need to specify in which time slot a message is transmitted. In block $t$, the $k$ th relay encodes an instance of $\mathcal{A}_{k}$ denoted by $\mathcal{A}_{k}^{t}=\left\{m_{k 1}^{t}, m_{k 2}^{t}, \ldots, m_{k n_{k}}^{t}\right\}$. The messages sent in block $t$ are parities of the messages sent in blocks $t-k$ to $t-1$. More precisely, if $m_{k i}$ is a child (direct parity) of $m_{l j}$ in the message tree, then in block $t$ we have

$$
m_{k i}^{t}=P_{\mathcal{B}_{k i}}\left(m_{l j}^{t-(k-l)}\right) .
$$

The set of all messages decoded by the $k$ th relay is specified by the set $\mathcal{D}_{k}$. The message set $\mathcal{A}_{k}$ is a descendent of $\mathcal{D}_{k}$. By decoding the messages in $\mathcal{D}_{k}$, the $k$ th relay knows all messages that are directly or indirectly parities of messages in $\mathcal{D}_{k}$, some of which are assigned to other relay terminals to be transmitted in subsequent blocks. For optimal encoding, the $k$ th relay should cooperate with other relay terminals to transmit messages known to the $k$ th relay. The $k$ th relay utilizes superposition broadcast encoding to encode multiple messages, which imposes limitations on the choice of $\mathcal{D}_{k}$ sets.

The $\mathcal{D}_{k}$ sets should have the following properties. First, the set $\mathcal{A}_{k}$ can be generated from $\mathcal{D}_{k}$, i.e., $\mathcal{D}_{k} \rightarrow \mathcal{A}_{k}$, while $\mathcal{D}_{k} \cap$ $\mathcal{A}_{k}=\{\}$. Second, a message and its parity cannot be both in $\mathcal{D}_{k}$, since the parity can be computed and thus removed from $\mathcal{D}_{k}$. Third, in order for the sets $\mathcal{D}_{k}$ to be consistent with the encoding method described in the next section, the $\mathcal{D}_{k}$ sets should be such that if a message $m_{l i}$ belongs to the set $\mathcal{D}_{k}$, then all messages of the same order $l$ and with a smaller second subscript should be a descendent of $\mathcal{D}_{k}$, i.e., $\forall i^{\prime} \leq i: \mathcal{D}_{k} \rightarrow m_{l i^{\prime}}$ (note that if $m_{r s} \in \mathcal{D}_{k}$ then $\mathcal{D}_{k} \rightarrow m_{r s}$ ). For the destination, we have $\mathcal{D}_{K+1}=\left\{m_{0}\right\}$. It should be noted that a collection of $\mathcal{D}_{k}$ sets satisfying these features always exists, since $\mathcal{D}_{k}=\left\{m_{0}\right\}, 1 \leq$ $k \leq K+1$, satisfies the above properties. (If $\mathcal{D}_{k}=\left\{m_{0}\right\}, 1 \leq$

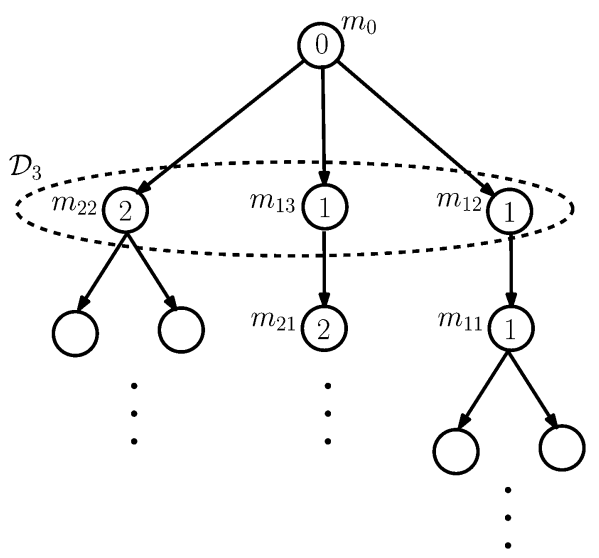

Fig. 7. Illustration of the properties of decoding sets.

$k \leq K+1$, then all the relays decode the source message. This choice of decoding sets gives the multihop DF rate, irrespective of the underlying message tree.)

To elaborate on the properties of $\mathcal{D}_{k}$ sets, consider the message tree in Fig. 7. In this example, $\mathcal{D}_{3}=\left\{m_{12}, m_{13}, m_{22}\right\}$ is consistent with all properties specified above. However, $\mathcal{D}_{3}=$ $\left\{m_{11}, m_{13}, m_{22}\right\}$ is invalid, because it violates the third property. The reason is that $m_{13}$ is encoded on top of $m_{11}$ and $m_{12}$ in the superposition encoding procedure described in the next section. Hence, decoding $m_{13}$ requires the decoding of $m_{11}$ and in particular $m_{12}$. But, $m_{12} \notin \mathcal{D}_{3}$ and $\mathcal{D}_{3} \nrightarrow m_{12}$. Thus, $\mathcal{D}_{3}=\left\{m_{11}, m_{13}, m_{22}\right\}$ does not completely specify all the messages decoded, violating the definition of the decoding set.

In summary, a parity forwarding protocol for a network with $K$ relays is defined by a three tuple $(\tau, \mathcal{A}, \mathcal{D})$ consisting of the message tree $\tau$, defining the relation between messages and parities; a partition $\mathcal{A}=\left\{\mathcal{A}_{0}, \mathcal{A}_{1}, \ldots, \mathcal{A}_{K}\right\}$ which assigns the messages to different relay terminals; and the set of decoding sets $\mathcal{D}=\left\{\mathcal{D}_{1}, \mathcal{D}_{2}, \ldots, \mathcal{D}_{K+1}\right\}$, which determines the set of messages each relay should decode.

\section{B. Encoding}

Messages are encoded using superposition coding. Consider as an example, the encoding of three messages $m_{a} \in\left\{1,2, \ldots, 2^{n R_{a}}\right\}, m_{b} \in\left\{1,2, \ldots, 2^{n R_{b}}\right\}$ $m_{c} \in\left\{1,2, \ldots, 2^{n R_{c}}\right\}$. First, $2^{n R_{c}}$ codewords $\mathbf{x}_{c}\left(m_{c}\right)$ are randomly generated according to $p\left(x_{c}\right)$. Next, $2^{n R_{b}}$ codewords $\mathbf{x}_{b}\left(m_{b} \mid m_{c}\right)$ are generated for each $\mathbf{x}_{c}$ codeword according to $p\left(x_{b} \mid x_{c}\right)$. We call the set $\left\{m_{c}\right\}$ the known message set and the set $\left\{\mathbf{x}_{c}\left(m_{c}\right) \mid m_{c}=1,2, \ldots, 2^{n R_{c}}\right\}$ the known codeword set for $m_{b}$, as the $\mathbf{x}_{b}$ codewords are generated for fixed known values of $\mathbf{x}_{c}\left(m_{c}\right)$. Similarly, $\mathbf{x}_{a}$ codewords are randomly generated for every pair of $m_{b}$ and $m_{c}$ messages by randomly choosing a codeword $\mathbf{x}_{c}\left(m_{a} \mid m_{b}, m_{c}\right)$ according to $p\left(x_{a} \mid x_{b}, x_{c}\right)$ conditioned upon fixed $\mathbf{x}_{b}\left(m_{b} \mid m_{c}\right)$ and $\mathbf{x}_{c}\left(m_{c}\right)$ codewords. Accordingly, the known message sets and codeword sets for $m_{a}$ are $\left\{m_{b}, m_{c}\right\}$ and $\left\{\left(\mathbf{x}_{b}\left(m_{b} \mid m_{c}\right), \mathbf{x}_{c}\left(m_{c}\right)\right) \mid m_{b}=\right.$ $\left.1, \ldots, 2^{n R_{b}}, m_{c}=1, \ldots, 2^{n R_{c}}\right\}$, respectively. The codeword $\mathbf{x}_{a}\left(m_{a} \mid m_{b}, m_{c}\right)$ encodes the three messages $m_{a}, m_{b}$, and $m_{c}$.

In the parity forwarding protocol, messages are encoded following the same procedure as in the above example using superposition coding. The key is to identify the known message sets 


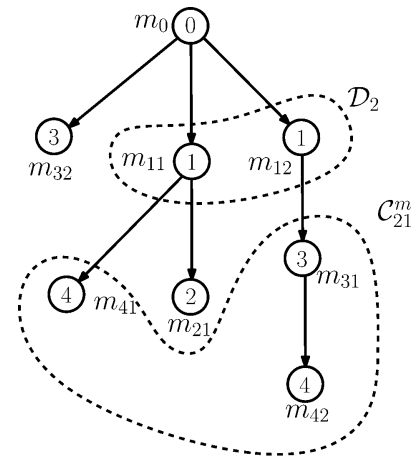

Fig. 8. Examples of known sets for the four-relay network in Fig. 6.

for each message $m_{k l}$ in the message tree to determine the set of all messages on top of which $m_{k l}$ should be encoded.

Definition 3 (Known Sets): The message $m_{k i}$ is superposed onto its known message sets $\mathcal{C}_{k i}^{m}$. First, within each $\mathcal{A}_{k}$, messages are superposed onto each other in the order of their second subscripts, i.e., at the $k$ th relay terminal, the message $m_{k i}$ is encoded on top of all messages $m_{k j}, j<i$. Furthermore, in each block, the $k$ th relay knows all messages of an order higher than $k$ which are descendants of $\mathcal{D}_{k}$, i.e., $\left\{m_{k^{\prime} i^{\prime}} \in \mathcal{M} \mid k^{\prime}>\right.$ $\left.k, \mathcal{D}_{k} \rightarrow m_{k^{\prime} i^{\prime}}\right\}$. Hence, the known message set of $m_{k i}$ is $\mathcal{C}_{k i}^{m}=\left\{m_{k j}, j<i\right\} \cup\left\{m_{k^{\prime} i^{\prime}} \in \mathcal{M} \mid k^{\prime}>k, \mathcal{D}_{k} \rightarrow m_{k^{\prime} l^{\prime}}\right\}$. Further, we use $\mathcal{C}_{k i}^{m}(t)$ to denote the instance of $\mathcal{C}_{k i}^{m}$ messages in the $t$ th block.

As an example of known sets consider Fig. 8 corresponding to the four-relay network in Fig. 5. Let $\mathcal{D}_{2}=\left\{m_{11}, m_{12}\right\}$ be the decoding set for the message $m_{21}$. Then, the known set $\mathcal{C}_{21}^{m}$ for $m_{12}$ consists of all descendants of $\mathcal{D}_{2}$ with orders higher than 2 , the order of $m_{21}$.

The next step in the generation of random codebooks is to assign a probability distribution and a random variable to each message in the tree. Let $X_{k i}$ represent the random variable corresponding to the encoding of $m_{k i}$. The set $\mathcal{C}_{k i}^{x}$ is defined as the set of random variables corresponding to messages in $\mathcal{C}_{k i}^{m}$, i.e., $\mathcal{C}_{k i}^{x}=\left\{X_{k i} \mid m_{k i} \in \mathcal{C}_{k i}^{m}\right\}$. Let $p\left(x_{k i} \mid \mathcal{C}_{k i}^{x}\right)$ be the conditional probability distribution associated with $X_{k i}$. Note that by properties of the decoding sets and known sets, $\prod_{k=0}^{K} \prod_{i=1}^{n_{k}} p\left(x_{k i} \mid \mathcal{C}_{k i}^{x}\right)$ is a valid joint probability distribution.

Random codebook construction starts with messages of the $K$ th relay that have empty known sets. For every message $m_{k i}, 1 \leq k \leq K, 1 \leq i \leq n_{i}$ with $\mathcal{C}_{k i}^{m}=\{\}, 2^{n R_{k i}}$ codewords $\mathbf{x}_{k i}\left(m_{k i}\right)$ are randomly generated according to the probability distribution $p\left(x_{k i}\right)$. In the next step, for every message $m_{k^{\prime} i^{\prime}}$ for which the codewords for all messages in the corresponding $\mathcal{C}_{k^{\prime} i^{\prime}}^{m}$ have already been constructed in previous steps, $2^{n R_{k^{\prime} i^{\prime}}}$ random codewords $\mathbf{x}_{k^{\prime} i^{\prime}}\left(m_{k^{\prime} i^{\prime}} \mid C_{k^{\prime} i^{\prime}}^{m}\right)$ are generated for every combination of codewords in $\mathcal{C}_{k^{\prime} \boldsymbol{i}^{\prime}}^{m}$ according to $p\left(x_{k^{\prime} \boldsymbol{i}^{\prime}} \mid \mathcal{C}_{k^{\prime} \boldsymbol{i}^{\prime}}^{x}\right)$. This procedure is repeated until random codebooks are generated for all messages. In block $t$, the $k$ th terminal, $0 \leq k \leq K$, transmits $\mathbf{x}_{k n_{k}}\left(m_{k n_{k}}^{t} \mid \mathcal{C}_{k n_{k}}^{m}(t)\right)$. This is equivalent to having $X_{k n_{k}}$ as the channel input by the $k$ th relay. For notational simplicity, let $X_{k} \triangleq X_{k n_{k}}, 0 \leq k \leq K$, so that the channel input by the $k$ th relay is represented by $X_{k}$.

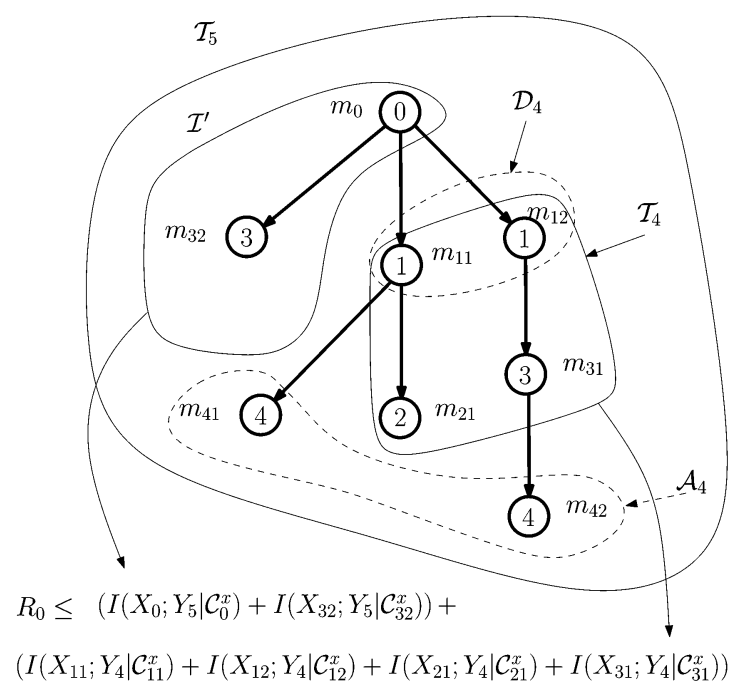

Fig. 9. An example of $\mathcal{D}_{4}, \mathcal{T}_{4}$, and $\mathcal{A}_{4}$ sets and their effect on the rate constraints. The rate constrain is derived by combining the constraints for $\mathcal{T}_{4}$ and $\mathcal{T}_{5}$

\section{Decoding}

Messages are decoded at each relay node and at the destination via joint decoding. A set of messages and their parity messages are jointly decoded by finding a combination of messages consistent with the parity relationship between messages, such that the corresponding codewords are jointly typical with the respective received sequences.

More specifically, in a parity forwarding protocol $(\tau, \mathcal{A}, \mathcal{D})$, the $k$ th relay needs to decode the set of messages $\mathcal{D}_{k}$. It does so by jointly decoding all messages in $\mathcal{D}_{k}$ together with all available parities of messages in $\mathcal{D}_{k}$. Since a parity message is available to node $k$ only if it is sent by a relay of order less than $k$, the joint decoding set $\mathcal{T}_{k}$ for the $k$ th relay is

$$
\mathcal{T}_{k}=\left\{m_{r s} \in \mathcal{M} \mid \mathcal{D}_{k} \rightarrow m_{r s}, r<k\right\} .
$$

Fig. 9 shows an example of $\mathcal{T}_{k}$ for $k=4$.

We are now ready to state the successful decoding condition for $\mathcal{T}_{k}$ for each node $k, 1 \leq k \leq K+1$ (recall that $k=K+1$ corresponds to the destination), which gives the main achievability rate for the parity-forwarding protocol:

Theorem 3 (Achievable Rate of Parity Forwarding): Consider a memoryless relay network with $K$ relay terminals defined by the probability distribution ${ }^{2}$

$$
p\left(y_{1}, y_{2}, \ldots, y_{K}, y_{K+1} \mid x_{0}, x_{1}, x_{2}, \ldots, x_{K}\right) .
$$

Using a parity forwarding $\operatorname{protocol}(\tau, \mathcal{A}, \mathcal{D})$, the source rate $R_{0}$ satisfying the following constraints maximized over the probability distribution $\prod_{\forall l, i: m_{l i} \in \mathcal{M}} p\left(x_{l i} \mid \mathcal{C}_{l i}^{x}\right)$ (along with a set of positive rates $R_{l p}$ ) is achievable:

$$
\begin{aligned}
& \sum_{\forall l, p: m_{l p} \in \mathcal{D}_{k}} R_{l p} \leq \sum_{\forall h j: m_{h j} \in \mathcal{I}^{\prime}} I\left(X_{h j} ; Y_{k} \mid \mathcal{C}_{h j}^{x}\right) \\
&+\sum_{\forall l^{\prime}, p^{\prime}: m_{l^{\prime} p^{\prime}} \in \mathcal{I}} R_{l^{\prime} p^{\prime}}
\end{aligned}
$$

${ }^{2}$ Recall that $X_{k} \triangleq X_{k n_{k}}, R_{k} \triangleq R_{k n_{k}}, 0 \leq k \leq K$. 
for all $1 \leq k \leq K+1$ and for all subsets $\mathcal{I}$ and $\mathcal{I}^{\prime}$ of $\mathcal{T}_{k}$ such that $\mathcal{I}$ contains no pairs of a message and its parity, i.e., $\forall m, m^{\prime} \in \mathcal{I} \subset \mathcal{T}_{k}: m \nrightarrow m^{\prime}$, and $\mathcal{I}^{\prime}=\left\{m \in \mathcal{I}_{k} \mid \mathcal{I} \nrightarrow m\right\}$.

Proof: The proof consists of a probability of error analysis for the joint decoding of $\mathcal{T}_{k}$ for each of the $k$ th receiver, $1 \leq$ $k \leq K+1$. This is done by using joint typicality test over all combinations of messages and their parities in $\mathcal{T}_{k}$ over a window of received sequences.

To identify the decoding window of received sequences at the $k$ th receiver, let $q$ be the smallest order sender (a relay or the source) of which a message is decoded by the $k$ th relay, i.e., $q$ is the smallest number such that $\exists l: m_{q l} \in \mathcal{D}_{k}$. Let $\mathbf{y}_{1}^{t}, \mathbf{y}_{2}^{t}, \ldots, \mathbf{y}_{K+1}^{t}$ denote the random sequences representing the received sequences in block $t$ at the first relay, the second relay, up to the destination, respectively. Then, according to (13), the decoding window for the $k$ th receiver (a relay or the destination) in block $t$ is of length $k-q$ and spans over blocks $t-(k-q)+1$ to $t$, i.e., decoding window is given by $\left(\mathbf{y}_{k}^{t-(k-q)+1}, \ldots, \mathbf{y}_{k}^{t}\right)$.

Without loss of generality, assume that some particular set of message values is transmitted. We first find the probability that for a particular $m_{l j}$, the receiver would incorrectly declare the codeword corresponding to some other message value to be jointly typical with the received sequence. Mathematically, for some message $m_{l j} \in \mathcal{T}_{k}$, the probability that its corresponding codeword $\mathbf{x}_{l j}\left(m_{l j} \mid \mathcal{C}_{l j}^{m}(t-(k-l)+1)\right), q \leq l<k$ is incorrectly declared jointly typical with $\mathbf{y}_{k}^{t-(k-l)+1}$, given $\mathcal{C}_{l j}^{x}(t-(k-$ $l)+1)$, is asymptotically bounded by $2^{-n I\left(X_{l j} ; Y_{k} \mid \mathcal{C}_{l j}^{x}\right)}$, where $\mathcal{C}_{l j}^{x}(t-(k-l)+1)$ denotes the set of codewords corresponding to messages in $\mathcal{C}_{l j}^{m}(t-(k-1)+1)$.

We will use the following fact to bound the probability of error for joint decoding over $\mathcal{T}_{k}$. The probability of incorrectly decoding at least one message in the joint decoding over $\mathcal{T}_{k}$ approaches zero as $n$ goes to infinity if for every subset $\mathcal{I}^{\prime} \subset$ $\mathcal{T}_{k}$, the probability that all messages in $\mathcal{I}^{\prime}$ are decoded incorrectly asymptotically approaches zero. This is similar to the bounding of the probability of decoding error in the multiple access channel; see ([8, Theorem 14.3.5]).

Now, for a particular set of transmitted message values in some fixed $\mathcal{I}^{\prime}$, the probability that the receiver would declare the codewords corresponding to some other set of valid combination of message values to be jointly typical with the respective received sequences over the decoding window is bounded by

$\prod_{\forall h, j: m_{h j} \in \mathcal{I}^{\prime}} 2^{-n I\left(X_{h j} ; Y_{k} \mid \mathcal{C}_{h j}^{x}\right)}$

$$
=2^{-n \sum_{\forall h, j: m_{h j} \in \mathcal{I}^{\prime}} I\left(X_{h j} ; Y_{k} \mid \mathcal{C}_{h j}^{x}\right)} .
$$

This fact will be used in calculating the overall probability of error by union bound. But first, we enumerate all possible subsets $\mathcal{I}^{\prime}$.

To enumerate all subsets $\mathcal{I}^{\prime}$ of incorrectly decoded messages, we take the following approach. Let $\mathcal{I}^{\prime} \in \mathcal{T}_{k}$ denote the set of incorrectly decoded messages. Let $\mathcal{J}=\left(\mathcal{I}^{\prime}\right)^{c}$, i.e., the set of correctly decoded messages. Note that if a message is decoded correctly, its parities must also be decoded correctly. Hence, $\mathcal{J}$ has the property that if a message is in $\mathcal{J}$, then all its parities in $\mathcal{T}_{k}$ must also be in $\mathcal{J}$. Since $\mathcal{J}$ contains the parities of all

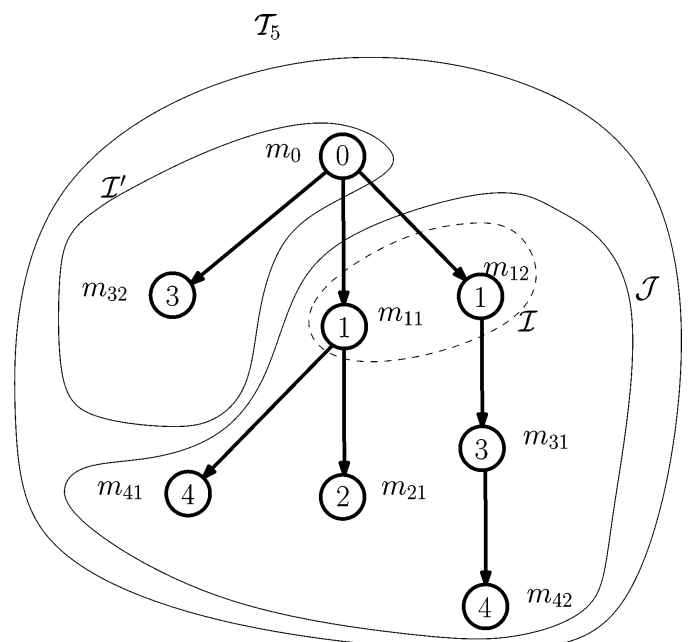

Fig. 10. An example of $\mathcal{I}, \mathcal{I}^{\prime}$, and $\mathcal{J}_{\mathcal{I}}$ subsets of $\mathcal{I}_{5}$, the set of all messages decoded at the destination. Fixing messages in $\mathcal{I}$, the number of valid combinations of messages in $\mathcal{I}^{\prime},\left|\mathcal{I}^{\prime}\right|$, is given by $\left|\mathcal{I}^{\prime}\right|=\left|\mathcal{I}_{5}\right| /|\mathcal{I}|$.

its messages, it must have a minimal generator, denoted here by $\mathcal{I}$. A minimal generator of $\mathcal{J}$ is a subset $\mathcal{I} \subset \mathcal{J}$ such that $\mathcal{I} \rightarrow \mathcal{J}$ and $\mathcal{I}$ contains no pairs of a message and its parity. (See Fig. 10, for an example.) Using this definition of $\mathcal{I}$, all subsets $\mathcal{I}^{\prime}$ of incorrectly decoded messages can be characterized via all subsets $\mathcal{I} \subset \mathcal{T}_{k}$ such that $\forall m, m^{\prime} \in \mathcal{I}: m \nrightarrow m^{\prime}$, and $\mathcal{I}^{\prime}=\left\{m \in \mathcal{T}_{k} \mid \mathcal{I} \nrightarrow m\right\}$.

Next, we enumerate all possible valid message value combinations ${ }^{3}$ in $\mathcal{I}^{\prime}$ that are consistent with message values in $\mathcal{J}$. Let $\left|\mathcal{I}^{\prime}\right|$ denote the number of valid combinations of message values in $\mathcal{I}^{\prime}$, while fixing the values of messages in $\left(\mathcal{I}^{\prime}\right)^{c}$. Note that since $\mathcal{J}=\left(\mathcal{I}^{\prime}\right)^{c}$, we have $\left|\mathcal{T}_{k}\right|=\left|\mathcal{I}^{\prime}\right| \cdot|\mathcal{J}|$.

Now, we relate $\left|\mathcal{T}_{k}\right|$ and $|\mathcal{J}|$ with message rates. Note that $\left|\mathcal{T}_{k}\right|$ and $|\mathcal{J}|$ are determined by the rates of messages in their respective minimal generators. This is because the values of the messages in the generator determine the values of all their parities. Now, the minimal generator of $\mathcal{T}_{k}$ is $\mathcal{D}_{k}$; the minimal generator of $\mathcal{J}$ is $\mathcal{I}$. Thus

$$
\begin{aligned}
& \frac{1}{n} \log \left|\mathcal{T}_{k}\right|=\sum_{\forall l, p: m_{l p} \in \mathcal{D}_{k}} R_{l p} \\
& \frac{1}{n} \log |\mathcal{J}|=\sum_{\forall l^{\prime}, p^{\prime}: m_{l^{\prime} p^{\prime}} \in \mathcal{I}} R_{l^{\prime} p^{\prime}}
\end{aligned}
$$

Finally, we return to the calculation of the probability of error. Fix some $\mathcal{I}$ and $\mathcal{I}^{\prime}$ as defined above, and fix a set of transmitted message values. By the union bound, the probability that all messages in the subset $\mathcal{I}^{\prime}$ are decoded incorrectly is bounded by the sum of the probabilities that the codewords corresponding to each incorrect set of valid combination of message values in $\mathcal{I}^{\prime}$ are declared jointly typical with the received sequences over the decoding window. Using (16), this probability of error is asymptotically bounded by

$$
\begin{aligned}
P_{e} & \leq\left|\mathcal{I}^{\prime}\right| 2^{-n \sum_{\forall h j: m_{h j} \in \mathcal{I}^{\prime}} I\left(X_{h j} ; Y_{k} \mid \mathcal{C}_{h j}^{x}\right)} \\
& =\frac{\left|\mathcal{T}_{k}\right|}{|\mathcal{J}|} 2^{-n \sum_{\forall h j: m_{h j} \in \mathcal{I}^{\prime}} I\left(X_{h j} ; Y_{k} \mid \mathcal{C}_{h j}^{x}\right)} .
\end{aligned}
$$

${ }^{3} \mathrm{~A}$ valid message value combination for a set of messages corresponds to a set of message values which are consistent with the relationships defined by the message tree. (See footnote 1 for an example.) 
Substituting (17) and (18) into the above, the upper bound asymptotically approaches zero if the following condition holds:

$$
\begin{aligned}
\underbrace{\sum_{\forall l, p: m_{l p} \in \mathcal{D}_{k}}}_{\frac{1}{n} \log \left|\mathcal{T}_{k}\right|} R_{l p} \leq \sum_{\forall h, j: m_{h j} \in \mathcal{I}^{\prime}} I\left(X_{h j} ; Y_{k} \mid \mathcal{C}_{h j}^{x}\right) \\
+\underbrace{\forall \sum_{l^{\prime}, p^{\prime}: m_{l^{\prime} p^{\prime}} \in \mathcal{I}} R_{l^{\prime} p^{\prime}}}_{\frac{1}{n} \log |\mathcal{J}|} .
\end{aligned}
$$

If this condition holds for every subset $\mathcal{I}$ and $\mathcal{I}^{\prime}$, then the probability of error for the joint decoding over $\mathcal{T}_{k}$ must approach zero asymptotically at $k$ th terminal. If this is true for all $k, 1 \leq k \leq$ $K+1$, then decoding must be successful for the overall parity forwarding protocol. This proves the theorem.

It should be noted that some of the inequalities in (15) may be redundant, allowing for further simplification of (15). In particular, an inequality in which there exists an $R_{l^{\prime} p^{\prime}}$ on the righthand side that does not appear on the left-hand side of another inequality may be ignored, since such an $R_{l^{\prime} p^{\prime}}$ is unbounded.

Fig. 9 describes an example of rate constraints produced by this theorem for the network in Fig. 5. The rate constraint on the source rate in Fig. 9 is derived by combining the two inequalities derived from (15) by setting $\mathcal{I}=\{\}$ for $k=4$, and $\mathcal{I}=\mathcal{D}_{4}$ for $k=5$.

\section{EXAmples of the PARITy ForWARding Protocols}

In this section, a number of examples of parity forwarding protocols are presented. These examples are chosen to illustrate different aspects of the parity forwarding scheme in a multiplerelay network and the conditions under which parity forwarding is capacity achieving. It is shown by example that previous DF rates are achieved by appropriately choosing the message tree, the message sets, and the decoding sets.

In the first example, it is demonstrated that the multihop DF rate is achievable using parity forwarding. This example introduces a simple form of a message tree: the chain message tree. The next two examples are also designed based on the chain message tree to demonstrate that the parity forwarding protocol improves the previous multihop DF rate. The difference between these three protocols also highlights the impact of selecting different decoding sets on the achieved rate. A new set of degradedness conditions are found under which the parity forwarding protocols in these examples are capacity achieving.

In addition, this section continues the two-relay example in Section III. For the two-relay network, a parity forwarding protocol that uses a message tree which is different from the chain message tree is described. While the chain message tree examples focus on the impact of the decoding sets on the achievable rate, this two-relay example illustrates the impact of the message tree on the achievable rate. Finally, this section concludes with a parity forwarding example for the single-relay channel to illustrate source message splitting.

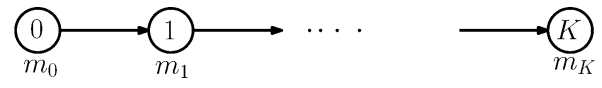

Fig. 11. Chain message tree.

\section{A. Achieving the Multihop Rate}

There are a number of parity forwarding protocols that achieve the multihop rate of [2]. ${ }^{4}$ A simple message tree that can be used to achieve the multihop rate is the chain message tree depicted in Fig. 11, in which each message is a parity for its parent message. Depending on which messages in the tree the relays decode or send, i.e., depending on the message sets and decoding sets, different parity forwarding protocols with different rates are obtained. The multihop rate can be achieved by having the source message $m_{0}$ decoded at all the relays.

Consider a network with a source and a destination and $K$ relays indexed by $k=1, \ldots, K$. In multihop parity forwarding, every relay $k$ decodes the source message $m_{0}$ in the chain message tree in Fig. 11, i.e., $\mathcal{D}_{k}=\left\{m_{0}\right\}$, and sends a parity message $m_{k}$ for the message of the $(k-1)$ th relay $m_{k-1}$, i.e., $\mathcal{A}_{k}=\left\{m_{k}\right\}$. The known message set at the $k$ th relay is given by $\mathcal{C}_{k}^{m}=\left\{m_{k+1}, m_{k+2}, \ldots, m_{K}\right\}$. Hence, the set $\mathcal{T}_{k}=\left\{m_{0}, m_{1}, \ldots, m_{k-1}\right\}$ should be jointly decoded at the $k$ th relay. Associating a random variable $X_{k}$ with the message $m_{k}$ in the message tree, (15) in Theorem 3 can be rewritten for this parity forwarding protocol as follows:

$$
\begin{aligned}
R_{0} \leq & \sum_{i=0}^{l-1} I\left(X_{i}, ; Y_{k} \mid X_{i+1}, \ldots, X_{K}\right)+R_{l} \\
= & I\left(X_{0}, X_{1}, \ldots, X_{l-1} ; Y_{k} \mid X_{l}, X_{l+1}, \ldots, X_{K}\right) \\
& +R_{l} \\
R_{0} \leq & \sum_{i=0}^{k-1} I\left(X_{i}, ; Y_{k} \mid X_{i+1}, \ldots, X_{K}\right) \\
= & I\left(X_{0}, X_{1}, \ldots, X_{k-1} ; Y_{k} \mid X_{k}, X_{k+1}, \ldots, X_{K}\right)
\end{aligned}
$$

for each $k, 1 \leq k \leq K+1$. Inequalities in (20a) are derived from (15) for $\mathcal{I}=\left\{m_{l}\right\}$ and $\mathcal{I}^{\prime}=\left\{m_{0}, m_{1}, \ldots, m_{l-1}\right\}$. Similarly, (20c) is derived from (15) for $\mathcal{I}=\{\}, \mathcal{I}^{\prime}=$ $\left\{m_{0}, m_{1}, \ldots, m_{k-1}\right\}$.

Now, note that in (20), all rates $R_{k}$ for $1 \leq k \leq K$ appear only on the right-hand side and thus are unbounded. Hence, (20) can be simplified by ignoring those inequalities with an $R_{k}$ on their right-hand side to achieve the following rate which is equal to the multihop rate of [2]:

$$
\begin{aligned}
R_{0} \leq I\left(X_{0}, X_{1}, \ldots, X_{k-1} ; Y_{k} \mid X_{k}, \ldots, X_{K}\right) & \\
& 1 \leq k \leq K+1 .
\end{aligned}
$$

${ }^{4}$ One such parity forwarding protocol is described in this section. Another parity forwarding scheme with the same rate can be devised, for example, by using a message tree in which the relay messages are independent direct parities of the source message. 


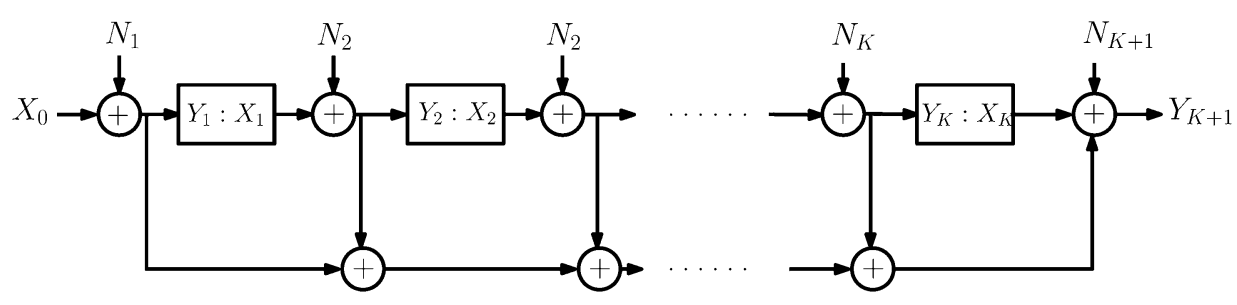

Fig. 12. A degraded chain network with additive noises $N_{k}, 1 \leq k \leq K$. Each relay can directly communicate only to its successor terminal. The $k$ th relay decodes the message of relay $k-1$ and forwards a parity for it.

\section{B. Short-Range Relays}

A different choice of decoded messages at the relays results in a different achievable rate in the previous example. For the chain message tree shown in Fig. 11, each relay may only decode the message of its predecessor, i.e., setting $\mathcal{D}_{k}=\left\{m_{k-1}\right\}$, and send a parity for $m_{k-1}$, i.e., $\mathcal{A}_{k}=\left\{m_{k}\right\}$ same as in the previous example. This would be a good scheme if each relay terminal has a small range and can only communicate to its successor relay (e.g., the degraded network shown in Fig. 12).

Theorem 3 can be used to give the achievable rate of this parity forwarding protocol. By setting $\mathcal{D}_{k}=\left\{m_{k-1}\right\}, 1 \leq k \leq$ $K$, the $k$ th relay in each block can compute parity messages with orders greater than $k$, i.e., $\mathcal{C}_{k}^{m}=\left\{m_{k+1}, m_{k+2}, \ldots, m_{K}\right\}$, $0 \leq k \leq K$. The only message with an order less than $k$ that is parity of $\mathcal{D}_{k}$ is $m_{k-1}$, hence, $\mathcal{T}_{k}=\left\{m_{k-1}\right\}, 0<k \leq K$, $1<k \leq K$. For the destination, $\mathcal{D}_{K+1}=\left\{m_{0}\right\}$, and $\mathcal{T}_{K+1}=$ $\left\{m_{0}, m_{1}, \ldots, m_{K}\right\}$.

Now, for $1 \leq k \leq K$, the only subsets $\mathcal{I}$ of $\mathcal{T}_{k}=\left\{m_{k-1}\right\}$ are \{\} and $\mathcal{T}_{k}$. Hence, the inequalities corresponding to $1 \leq k \leq K$ in (15) are given by

$$
\begin{gathered}
R_{k-1} \leq I\left(X_{k-1} ; Y_{k} \mid X_{k}, \ldots, X_{K}\right) \quad: \mathcal{I}=\{\}, \\
\mathcal{I}^{\prime}=\left\{m_{k-1}\right\} \\
R_{k-1} \leq R_{k-1} \quad: \mathcal{I}=\left\{m_{k-1}\right\}, \mathcal{I}^{\prime}=\{\} .
\end{gathered}
$$

Finally, since $\mathcal{T}_{K+1}=\left\{m_{0}, m_{1}, \ldots, m_{K}\right\}$ and $\mathcal{D}_{K+1}=$ $\left\{m_{0}\right\}$, (15) gives the following inequalities for $k=K+1$ :

$$
\begin{aligned}
R_{0} \leq \sum_{i=0}^{l-1} I\left(X_{i} ; Y_{K+1} \mid X_{i+1}, \ldots, X_{K}\right)+R_{l} \\
1 \leq l \leq K \\
R_{0} \leq \sum_{i=0}^{K} I\left(X_{i} ; Y_{K+1} \mid X_{i+1}, \ldots, X_{K}\right) .
\end{aligned}
$$

The above inequalities are derived by setting $\mathcal{I}=\left\{m_{l}\right\}$ and $\mathcal{I}^{\prime}=\left\{m_{0}, \ldots, m_{l-1}\right\}$ for $1 \leq l \leq K$, and $\mathcal{I}=\{\}$ for $l=$ $K+1$.

Using the chain rule for mutual information and substituting the constraints on $R_{l}$ for $1 \leq l \leq K$ in (23) results in an achievable rate for this network specified in the next theorem.

Theorem 4: For a memoryless $K$-relay network defined by $p\left(y_{1}, y_{2}, \ldots, y_{K+1} \mid x_{0}, x_{1}, \ldots, x_{K}\right)$, fixing any $p\left(x_{0}, x_{1}, \ldots, x_{K}\right)$, the source rate $R_{0}$ satisfying the following constraints is achievable:

$$
R_{0}<I\left(X_{0} ; Y_{1} \mid X_{1}, X_{2}, \ldots, X_{K}\right)
$$

$$
\begin{aligned}
R_{0}< & I\left(X_{0} ; Y_{K+1} \mid X_{1}, \ldots, X_{K}\right) \\
& +I\left(X_{1} ; Y_{2} \mid X_{2}, \ldots, X_{K}\right) \\
R_{0}< & I\left(X_{0}, X_{1} ; Y_{K+1} \mid X_{2}, \ldots, X_{K}\right) \\
& +I\left(X_{2} ; Y_{3} \mid X_{3}, \ldots, X_{K}\right) \\
\vdots & \\
R_{0}< & I\left(X_{0}^{K-2} ; Y_{K+1} \mid X_{K-1}, X_{K}\right) \\
& +I\left(X_{K-1} ; Y_{K} \mid X_{K}\right) \\
R_{0}< & I\left(X_{0}^{K} ; Y_{K+1}\right)
\end{aligned}
$$

where $X_{i}^{j} \triangleq\left(X_{i}, X_{i+1}, \ldots, X_{j}\right)$. Further, the above rate maximized over $p\left(x_{0}, x_{1}, \ldots, x_{K}\right)$ is the capacity of this network if $X_{k}-\left(Y_{k+1}, X_{k+1}^{K}\right)-Y_{k+2}^{K+1}$ and $X_{0}^{k-1}-\left(Y_{K+1}, X_{k}^{K}\right)-Y_{k+1}^{K}$ form Markov chains.

Proof: The achievability follows from the statements leading to the theorem. The converse can be proved using the cut-set bound [21, Theorem 14.10.1].

The cut-set bound states that the source rate $R_{0}$ is upper bounded by the following inequalities for $0 \leq k \leq K$ :

$$
R_{0}<I\left(X_{0}, \ldots, X_{k} ; Y_{k+1}, \ldots, Y_{K+1} \mid X_{k+1}, \ldots, X_{K}\right)
$$

for some $p\left(x_{0}, x_{1}, \ldots, x_{K}\right)$. The above upper bound coincides with the achievable rate in (24) if $X_{k}-\left(Y_{k+1}, X_{k+1}^{K}\right)-Y_{k+2}^{K+1}$ and $X_{0}^{k-1}-\left(Y_{K+1}, X_{k}^{K}\right)-Y_{k+1}^{K}$ form Markov chains. This can be proved by expanding (25) as follows:

$$
\begin{aligned}
& I\left(X_{0}^{k} ; Y_{k+1}^{K+1} \mid X_{k+1}^{K}\right) \\
& \quad \stackrel{(a)}{=} I\left(X_{k} ; Y_{k+1}^{K+1} \mid X_{k+1}^{K}\right)+I\left(X_{0}^{k-1} ; Y_{k+1}^{K+1} \mid X_{k}^{K}\right) \\
& \quad \stackrel{(b)}{=} I\left(X_{k} ; Y_{k+1} \mid X_{k+1}^{K}\right)+I\left(X_{0}^{k-1} ; Y_{k+1}^{K+1} \mid X_{k}^{K}\right) \\
& \quad \stackrel{(c)}{=} I\left(X_{k} ; Y_{k+1} \mid X_{k+1}^{K}\right)+I\left(X_{0}^{k-1} ; Y_{K+1} \mid X_{k}^{K}\right)
\end{aligned}
$$

where (a) follows from the chain rule for mutual information, (b) holds since $X_{k}-\left(Y_{k+1}, X_{k+1}^{K}\right)-Y_{k+2}^{K+1}$ is a Markov chain, and (c) holds since $X_{0}^{k-1}-\left(Y_{K+1}, X_{k}^{K}\right)-Y_{k+1}^{K}$ is a Markov chain. Fig. 12 shows an example of such a degraded network with additive noise at receivers.

Intuitively, each rate constraint in the above achievable rate consists of two components: the $I\left(X_{k} ; Y_{k+1} \mid X_{k+1}^{K}\right)$ component represents the transferable information from the $k$ th relay terminal to its successor, and the other component $I\left(X_{0}^{k-1} ; Y_{K+1} \mid X_{k}^{K}\right)$ corresponds to the rate at which source and the first $k-1$ relays can cooperatively communicate to the destination. The degradedness conditions in Theorem 4 ensure 


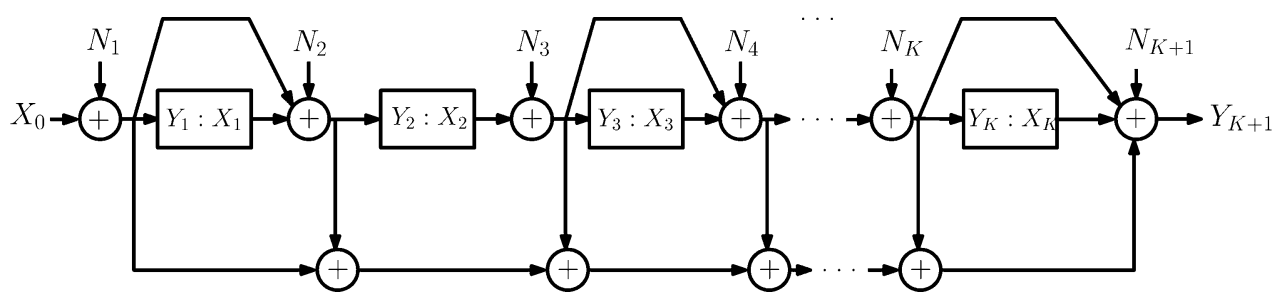

Fig. 13. A degraded chain network with additive noise. For odd $k$ 's, the $k$ th relay, $1 \leq k \leq K$, helps relay $k+1$ decode the message of relay $k-1$.

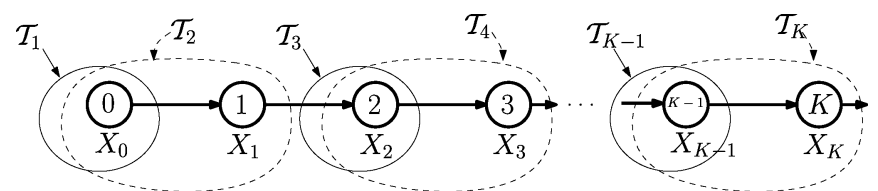

Fig. 14. The sets $\mathcal{T}_{k}$ for the example in Fig. 13.

that the achievable rate coincides with the cut-set defined at the $k$ th relay separating the source and the first $k$ relays from the destination and the rest of the relays.

The rate achieved by this protocol can be higher than the multihop rate if relays have a short range and the channel from the source to relays that are far away is blocked. This example generalizes Protocol B introduced in Section III. It demonstrates that the best choice of decoding sets at the relays depends on the network condition. In general, the best achievable parity forwarding rate is obtained by searching through all possible parity forwarding protocols for the network.

\section{Coupled Relays}

It is possible to group the relays to cooperate in the previous example. The example in this section describes a parity forwarding protocol based on the same chain message tree introduced in the previous example, but in which the relays are coupled in groups of two, cooperatively communicating to the next relay. In the network shown in Fig. 13, the source and the first relay cooperatively communicate to the second relay. The second relay and the third relay cooperatively communicate to the fourth relay, and so on. Assume that the number of relays $K$ is odd. Similar to the previous examples the $k$ th relay sends a parity message for the message of relay $k-1$, i.e., $\mathcal{A}_{k}=\left\{m_{k}\right\}$. The difference as compared to the previous examples, is the choice of messages that are decoded at relays. For odd $k$ 's, the $k$ th relay decodes the message of relay $k-1$, and for even $k$ 's, it decodes the message of relay $k-2$. This scenario corresponds to setting $\mathcal{D}_{k}=\left\{m_{k-1}\right\}$ for odd $k$, and $\mathcal{D}_{k}=\left\{m_{k-2}\right\}$ for even $k$. Hence, $\mathcal{T}_{k}=\left\{m_{k-1}\right\}$ for odd $k$, and $\mathcal{T}_{k}=\left\{m_{k-2}, m_{k-1}\right\}$ for even $k$ (see Fig. 14). For the destination, $\mathcal{T}_{K+1}=\left\{m_{0}, \ldots, m_{K}\right\}$, and $\mathcal{D}_{K+1}=\left\{m_{0}\right\}$. The following theorem specifies an achievable rate for this setting.

Theorem 5: For a memoryless $K$-relay network, $K$ odd, defined by $p\left(y_{1}, y_{2}, \ldots, y_{K+1} \mid x_{0}, x_{1}, \ldots, x_{K}\right)$, fixing any $p\left(x_{0}, x_{1}, \ldots, x_{K}\right)$, the source rate $R_{0}$ satisfying the following constraints is achievable:

$$
R_{0}<I\left(X_{0} ; Y_{1} \mid X_{1}^{K}\right)
$$

$$
\begin{aligned}
R_{0}< & I\left(X_{0}, X_{1} ; Y_{2} \mid X_{2}^{K}\right) \\
R_{0}< & I\left(X_{2} ; Y_{3} \mid X_{3}^{K}\right) \\
& +I\left(X_{0}, X_{1} ; Y_{K+1} \mid X_{2}^{K}\right) \\
R_{0}< & I\left(X_{2}, X_{3} ; Y_{4} \mid X_{4}^{K}\right) \\
& +I\left(X_{0}, X_{1} ; Y_{K+1} \mid X_{2}^{K}\right) . \\
\vdots & \\
R_{0}< & I\left(X_{K-2} ; Y_{K-1} \mid X_{K-1}, X_{K}\right) \\
& +I\left(X_{0}^{K-3} ; Y_{K+1} \mid X_{K-2}^{K}\right) \\
R_{0}< & I\left(X_{K-2}^{K-1} ; Y_{K} \mid X_{K}\right) \\
& +I\left(X_{0}^{K-3} ; Y_{K+1} \mid X_{K-2}^{K}\right) \\
R_{0}< & I\left(X_{0}, X_{1}, \ldots, X_{K} ; Y_{K+1}\right) .
\end{aligned}
$$

Further, the above rate maximized over $p\left(x_{0}, x_{1}, \ldots, x_{K}\right)$ is the capacity of this network if $X_{k}-\left(Y_{k+1}, X_{k+1}^{K}\right)-Y_{k+2}^{K}$ and $X_{0}^{k-1}-\left(Y_{K+1}, X_{k}^{K}\right)-Y_{k+1}^{K}$ are Markov chains for $k$ even, and $\left(X_{k-1}, X_{k}\right)-\left(Y_{k+1}, X_{k+1}^{K}\right)-Y_{k+2}^{K+1}$ and $X_{0}^{k-2}-$ $\left(Y_{K+1}, X_{k-1}^{K}\right)-Y_{k+1}^{K}$ form Markov chains for $k$ odd.

Proof: This rate is derived using (15) as follows. For $k$ odd, $\mathcal{T}_{k}=\left\{m_{k-1}\right\}$ has only two subsets \{\} and $\mathcal{T}_{k}$. Thus, (15) for odd $k, 1 \leq k \leq K$, results in

$$
\begin{aligned}
R_{k-1} & \leq I\left(X_{k-1} ; Y_{k} \mid X_{k}, \ldots, X_{K}\right) \quad: \mathcal{I}=\{\} \\
\mathcal{I}^{\prime} & =\left\{m_{k-1}\right\} \\
R_{k-1} & \leq R_{k-1}: \mathcal{I}=\left\{m_{k-1}\right\}, \mathcal{I}^{\prime}=\{\} .
\end{aligned}
$$

For even $k, \mathcal{T}_{k}=\left\{m_{k-2}, m_{k-1}\right\}$ which has subsets \{\}$,\left\{m_{k-2}\right\},\left\{m_{k-1}\right\}$, and $\mathcal{T}_{k}$. Consequently, the corresponding constraints in (15) for even $k, 2 \leq k<K$, state that for $\mathcal{I}=\{\}, \mathcal{I}^{\prime}=\mathcal{T}_{k}$

$$
\begin{aligned}
& R_{k-2} \leq I\left(X_{k-2} ; Y_{k} \mid X_{k-1}, \ldots, X_{K}\right) \\
& \quad+I\left(X_{k-1} ; Y_{k} \mid X_{k}, \ldots, X_{K}\right)
\end{aligned}
$$

for $\mathcal{I}=\left\{m_{k-2}\right\}, \mathcal{I}^{\prime}=\left\{m_{k-2}\right\}$

$$
R_{k-2} \leq I\left(X_{k-2} ; Y_{k} \mid X_{k-1}, \ldots, X_{K}\right)+R_{k-1}
$$

and for $\mathcal{I}=\mathcal{T}_{k}, \mathcal{I}^{\prime}=\{\}$ :

$$
R_{k-2} \leq R_{k-2}
$$

Finally, the rate constraints at the destination are also given by a set of inequalities given in (23). The rate given in (27) is obtained by using the chain rule for mutual information and ignoring constraints that have a rate $R_{k}$ for an odd $k$ on their 
right-hand sides, as only $R_{k}$ 's with an even $k$ are constrained by the above set of bounds.

The converse is proved using the cut-set bound. For even $k$ 's, $1 \leq k \leq K$, the cut-set bound results in a set of upper bounds similar to those in (25) which are achievable using this protocol if for $k$ even, $0 \leq k \leq K-1, X_{k}-\left(Y_{k+1}, X_{k+1}^{K}\right)-Y_{k+2}^{K}$ and $X_{0}^{k-1}-\left(Y_{K+1}, X_{k}^{K}\right)-Y_{k+1}^{K}$ are Markov chains. For odd $k$ 's, the cut-set bound coincides with (27) if $\left(X_{k-1}, X_{k}\right)-$ $\left(Y_{k+1}, X_{k+1}^{K}\right)-Y_{k+2}^{K+1}$ and $X_{0}^{k-2}-\left(Y_{K+1}, X_{k-1}^{K}\right)-Y_{k+1}^{K}$ form Markov chains. This is proved in the following:

$$
\begin{gathered}
\stackrel{(a)}{=} I\left(X_{k-1}, X_{k} ; Y_{k+1}^{K+1} \mid X_{k+1}^{K}\right) \\
\quad+I\left(X_{0}^{k-2} ; Y_{k+1}^{K+1} \mid X_{k-1}^{K}\right) \\
\stackrel{(b)}{=} I\left(X_{k-1}, X_{k} ; Y_{k+1} \mid X_{k+1}^{K+1}\right) \\
\quad+I\left(X_{0}^{k-2} ; Y_{k+1}^{K+1} \mid X_{k-1}^{K}\right) \\
\stackrel{(c)}{=} I\left(X_{k-1}, X_{k} ; Y_{k+1} \mid X_{k+1}^{K+1}\right) \\
\quad+I\left(X_{0}^{k-2} ; Y_{K+1} \mid X_{k-1}^{K}\right)
\end{gathered}
$$$$
R_{0}<I\left(X_{0}, \ldots, X_{k} ; Y_{k+1}, \ldots, Y_{K+1} \mid X_{k+1}, \ldots, X_{K}\right)
$$

where (a) follows from the chain rule for mutual information, (b) holds since $\left(X_{k-1}, X_{k}\right)-\left(Y_{k+1}, X_{k+1}^{K}\right)-Y_{k+2}^{K+1}$ forms a Markov chain for odd $k, 1 \leq k \leq K$, and (c) holds since $X_{0}^{k-2}-\left(Y_{K+1}, X_{k-1}^{K}\right)-Y_{k+1}^{K}$ is a Markov chain.

An example of such a degraded network is shown in Fig. 13. For this network, (27) achieves the cut-set bound if we consider the cut-set defined at the $k$ th relay, separating the source and the first $k$ relays from the destination and relays $k+1$ up to $K$. For an odd $k$, the rate of this cut-set equals to the cooperative information rate from relays $k-1$ and $k$ to relay $k+1$, plus the cooperative rate from the source and relays 1 up to $k-2$ to the destination. For an even $k$, the rate of this cut-set is given by the rate at which the $k$ th terminal can communicate to relay $k+1$, plus the rate at which the source and the first $k-1$ relays can communicate to the destination.

\section{Two-Relay Network With a Mixture of Semideterministic and Deterministic Subnetworks}

The previous examples explain the effect of decoding different messages at relays on the achieved rate. The form of the message tree also affects the achievable rate of parity forwarding. In this example another parity forwarding protocol is introduced for the two-relay network of Section III with a different type of message tree.

Consider the two-relay network defined in Section III. A parity forwarding protocol can be devised for this network based on the message tree shown in Fig. 15. In this parity forwarding protocol, the first relay decodes the source message and transmits two independent parity messages for the source message. The second relay decodes one of the two parity messages sent by the first relay.

More precisely, we have $\mathcal{A}_{0}=\left\{m_{0}\right\}, \mathcal{A}_{1}=$ $\left\{m_{11}, m_{12}\right\}, \mathcal{A}_{2}=\left\{m_{21}\right\}$. The first relay decodes $m_{0}$, i.e., $\mathcal{D}_{1}=\left\{m_{0}\right\}$, and the second relay decodes $m_{11}$, i.e., $\mathcal{D}_{2}=\left\{m_{11}\right\}$. Consequently, the source knows all

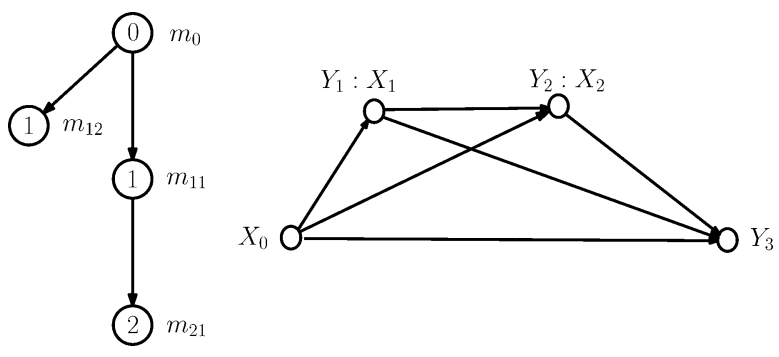

Fig. 15. Message tree for two-relay network with semideterministic channel from the first relay to the second relay.

other messages, and the first relay knows $m_{21}$. Hence, $\mathcal{C}_{0}^{x}=\left\{m_{11}, m_{12}, m_{21}\right\}, \mathcal{C}_{11}^{x}=\left\{m_{21}\right\}, \mathcal{C}_{12}^{x}=\left\{m_{11}, m_{21}\right\}$ (note that $m_{11}$ is in the known set of $m_{12}$, since superposition encoding is used for encoding $m_{12}$ on top of $m_{11}$ and $m_{21}$ ), and $\mathcal{C}_{21}^{x}=\{\}$. Since $\mathcal{D}_{1}=\left\{m_{0}\right\}$ and $\mathcal{A}_{1}=\left\{m_{11}, m_{12}\right\}$, we have $\mathcal{T}_{1}=\left\{m_{0}\right\}$. Similarly, since $\mathcal{D}_{2}=\left\{m_{11}\right\}$ and $\mathcal{A}_{2}=\left\{m_{21}\right\}$, we have $\mathcal{T}_{2}=\left\{m_{11}\right\}$. At the destination $\mathcal{D}_{3}=\left\{m_{0}\right\}$, and $\mathcal{T}_{3}=\left\{m_{0}, m_{11}, m_{12}, m_{21}\right\}$. Associating random variables $X_{0}, X_{11}, X_{12}, X_{21} \quad$ with messages $m_{0}, m_{11}, m_{12}, m_{21}$, respectively, Theorem 3 gives the following achievable rate for an arbitrary two-relay network under any fixed distribution $p\left(x_{0}, x_{11}, x_{12}, x_{21}\right)$ (the detailed derivation is omitted)

$$
\begin{aligned}
& R_{0}<I\left(X_{0} ; Y_{1} \mid X_{11}, X_{12}, X_{21}\right) \\
& R_{0}<I\left(X_{0}, X_{12} ; Y_{3} \mid X_{11}, X_{21}\right)+I\left(X_{11} ; Y_{2} \mid X_{21}\right) \\
& R_{0}<I\left(X_{0}, X_{11}, X_{12}, X_{21} ; Y_{3}\right)
\end{aligned}
$$

The above rate can be shown to be the capacity of a two-relay network if the channel from the source to the first relay is stronger than the channel from the source to the second relay and the destination, and the channel from the first relay to the second relay is semideterministic [10].

Intuitively, this protocol is suitable for this network if we recall that partial decoding at the relay is the optimal strategy for the semideterministic single-relay channel. In this channel, the capacity-achieving strategy is for the source to split its message into two messages [10]; the relay decodes one of them and forwards a parity message for it. Similar to the single-relay case, in a multirelay network with a semideterministic channel from the first relay to the second relay, the first relay should split its message into two parts; the second relay only partially decodes the message of the first relay. This is optimal as shown in the next theorem.

Theorem 6: The capacity of a two-relay network defined by $p\left(y_{1}, y_{2}, y_{3} \mid x_{0}, x_{1}, x_{2}\right)$ in which $X_{0}-\left(X_{1}, X_{2}, Y_{1}\right)-Y_{3}$ is a Markov chain, and the channel from the first relay to the second relay is semideterministic, i.e., $Y_{2}=f\left(X_{1}, X_{2}\right)$ for a deterministic function $f(\cdot, \cdot)$, is given by

$$
\begin{aligned}
R_{0}< & I\left(X_{0} ; Y_{1} \mid X_{1}, X_{2}\right) \\
R_{0}< & I\left(X_{0} ; Y_{3} \mid X_{1}, X_{2}\right)+I\left(X_{1} ; Y_{3} \mid Y_{2}, X_{2}\right) \\
& \quad+H\left(Y_{2} \mid X_{2}\right) \\
R_{0}< & I\left(X_{0}, X_{1}, X_{2} ; Y_{3}\right)
\end{aligned}
$$

maximized over $p\left(x_{0}, x_{1}, x_{2}\right)$. 
Proof: For achievability, setting $X_{0}=X_{0}, X_{1}=X_{12}$, $X_{2}=X_{21}$, and $X_{11}=Y_{2}$ reduces (30) to (31). Note that $X_{11}$ is a deterministic function of $X_{12}$ and $X_{21}$. Using this fact, the derivation of (31a) and (31c) is straightforward. The achievability of (31b) is proved in the following:

$$
\begin{aligned}
& I\left(X_{0}, X_{12} ; Y_{3} \mid X_{11}, X_{21}\right)+I\left(X_{11} ; Y_{2} \mid X_{21}\right) \\
& \stackrel{(\mathrm{a})}{=} I\left(X_{0} ; Y_{3} \mid X_{11}, X_{12}, X_{21}\right) \\
& \quad+I\left(X_{12} ; Y_{3} \mid X_{11}, X_{21}\right)+I\left(X_{11} ; Y_{2} \mid X_{21}\right) \\
& \stackrel{(\mathrm{b})}{=} I\left(X_{0} ; Y_{3} \mid X_{12}, X_{21}\right) \\
& \quad+I\left(X_{12} ; Y_{3} \mid X_{11}, X_{21}\right)+I\left(Y_{2} ; Y_{2} \mid X_{2}\right) \\
& \stackrel{(\mathrm{c})}{=} I\left(X_{0} ; Y_{3} \mid X_{1}, X_{2}\right) \\
& \quad+I\left(X_{1} ; Y_{3} \mid Y_{2}, X_{2}\right)+H\left(Y_{2} \mid X_{2}\right),
\end{aligned}
$$

where (a) follows from the chain rule for mutual information, (b) follows since $X_{11}$ is a function of $X_{12}$ and $X_{21}$, and (c) follows from $I\left(Y_{2} ; Y_{2} \mid X_{2}\right)=H\left(Y_{2} \mid X_{2}\right)$.

To prove the converse, we use the cut-set bound (10). The Markov chain $X_{0}-\left(X_{1}, X_{2}, Y_{1}\right)-Y_{3}$ along with the condition $Y_{2}=f\left(X_{1}, X_{2}\right)$ leads to the Markov chain $X_{0}-\left(X_{1}, X_{2}, Y_{1}\right)-$ $\left(Y_{2}, Y_{3}\right)$ for this network. Hence, the bound (10a) coincides with (31a). The bound (10c) is also equivalent to (31c). It remains to prove that (31b) is achievable and meets the cut-set bound (10b). This can be proved by expanding (10b) as follows:

$$
\begin{aligned}
R_{0}< & I\left(X_{0}, X_{1} ; Y_{2}, Y_{3} \mid X_{2}\right) \\
= & I\left(X_{0} ; Y_{2}, Y_{3} \mid X_{1}, X_{2}\right)+I\left(X_{1} ; Y_{2}, Y_{3} \mid X_{2}\right) \\
= & I\left(X_{0} ; Y_{3} \mid X_{1}, X_{2}, Y_{2}\right)+I\left(X_{0} ; Y_{2} \mid X_{1}, X_{2}\right) \\
& +I\left(X_{1} ; Y_{3} \mid X_{2}, Y_{2}\right)+I\left(X_{1} ; Y_{2} \mid X_{2}\right) \\
= & I\left(X_{0} ; Y_{3} \mid X_{1}, X_{2}, Y_{2}\right) \\
& +I\left(X_{1} ; Y_{3} \mid X_{2}, Y_{2}\right)+H\left(Y_{2} \mid X_{2}\right)
\end{aligned}
$$

since $H\left(Y_{2} \mid X_{1}, X_{2}\right)=0$ and $I\left(X_{0} ; Y_{2} \mid X_{1}, X_{2}\right)=0$ for $Y_{2}=f\left(X_{1}, X_{2}\right)$.

In addition to the two parity forwarding protocols described in Section III and the above example, there are other possible parity forwarding protocols for a two-relay network as well. For example, the source message may also be split to allow partial decoding at the first relay as well. The next example illustrates splitting the source message in the single-relay network.

\section{E. Generalized Decode-and-Forward}

In this section, the single-relay channel is used as an example to illustrate source message splitting in the parity-forwarding frameworks. In this example, the source may send two messages $m_{01}, m_{0}$. The message $m_{01}$ is a random bin index for $m_{0}$. This is equivalent to splitting $m_{0}$ into two parts. The relay may only decode $m_{01}$ which is of a lower rate. This strategy, which is similar $^{5}$ to the generalized DF ([8, Theorem 7]), increases the DF rate for example in semideterministic relay channel [10] or a relay channel with an orthogonal source-relay channel [22].

\footnotetext{
${ }^{5}$ The scheme described here where the source message split using a parity message is slightly different from the generalized DF approach of ([8, Theorem 7]), where the two source messages are independent of each other. However, the two schemes result in the same rate.
}

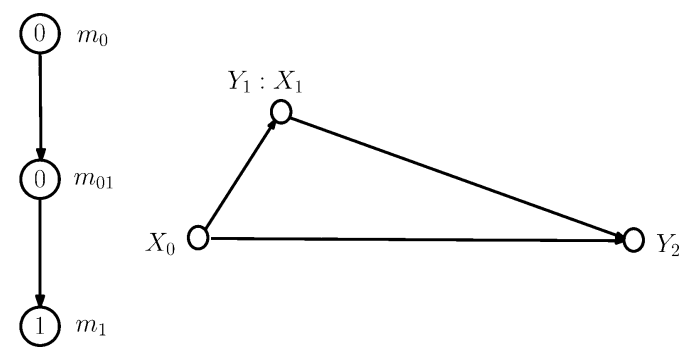

Fig. 16. The message tree for single-relay generalized decode-and-forward.

The message tree used for this single-relay network is shown in Fig. 16, which is similar to the message tree for the tworelay examples in Section III. The difference lies in the way the messages are associated with the source and the relay for the single-relay network. In this case, the message sets are defined as follows. The source sends both $m_{0}$ and $m_{01}$, i.e., $\mathcal{A}_{0}=$ $\left\{m_{0}, m_{01}\right\}$. The relay decodes only $m_{01}$, i.e., $\mathcal{D}_{1}=\left\{m_{01}\right\}$, and sends a parity for $m_{01}$, i.e., $\mathcal{A}_{1}=\left\{m_{1}\right\}$. Thus, the known message sets for each message are given by $\mathcal{C}_{1}^{m}=\{\}, \mathcal{C}_{01}^{m}=\left\{m_{1}\right\}$, and $\mathcal{C}_{0}^{m}=\left\{m_{1}, m_{01}\right\}$. Superposition encoding is used to encode messages at the source and at the relay by associating the random variables $X_{1}, X_{01}$, and $X_{0}$ with messages $m_{1}, m_{01}$, and $m_{0}$, respectively. The relay encodes $m_{1}$ by forming a random codebook of size $2^{n R_{1}}$. The source first encodes $m_{01}$ superimposed on $m_{1}$, and then superimposes $m_{0}$ on top of $m_{01}$ and $m_{1}$.

Theorem 3 gives the following rate constraints for this protocol. At the relay,

$$
R_{01} \leq I\left(X_{01} ; Y_{1} \mid X_{1}\right)
$$

and at the destination

$$
\begin{aligned}
R_{0} \leq & I\left(X_{0} ; Y_{2} \mid X_{01}, X_{1}\right)+R_{01} \\
R_{0} \leq & I\left(X_{0} ; Y_{2} \mid X_{01}, X_{1}\right)+I\left(X_{01} ; Y_{2} \mid X_{1}\right)+R_{1} \\
R_{0} \leq & I\left(X_{0} ; Y_{2} \mid X_{01}, X_{1}\right) \\
& +I\left(X_{01} ; Y_{2} \mid X_{1}\right)+I\left(X_{1} ; Y_{2}\right) .
\end{aligned}
$$

The above can be simplified by using the chain rule for mutual information and ignoring (34c) because $R_{1}$ is unbounded. Thus, Theorem 3 gives the following rate maximized over $p\left(x_{0}, x_{01}, x_{1}\right)$ for this protocol:

$$
\begin{aligned}
R_{0} & \stackrel{(a)}{\leq} I\left(X_{0} ; Y_{2} \mid X_{01}, X_{1}\right)+I\left(X_{01} ; Y_{1} \mid X_{1}\right) \\
R_{0} & \stackrel{(b)}{\leq} I\left(X_{0}, X_{01}, X_{1} ; Y_{2}\right) \\
& \stackrel{(c)}{=} I\left(X_{0}, X_{1} ; Y_{2}\right)
\end{aligned}
$$

where (a) follows by combining (34a) and (34b), (b) follows by applying the chain rule for mutual information to (34d), and (c) follows from $X_{01}-\left(X_{0}, X_{1}\right)-Y_{2}$ as $X_{01}$ can only affect $Y_{2}$ through the channel inputs $X_{0}$ and $X_{1}$. Note that the rate in (35) is equal to the rate achieved by the generalized decode-andforward method (also known as partial decode-and-forward) of ([8, Theorem 7]). 


\section{CONCLUSION}

This paper formulates a class of DF strategies, called parity forwarding, for an arbitrary multirelay network. In this set of strategies, the relay nodes forward bin indices for the messages of other transmitters. A message tree structure is utilized to characterize the encoding and decoding procedures and the dependencies between messages and their bin indices. Parity forwarding improves previous DF strategies because of its flexibility. Further, it achieves the capacities of new types of degraded multirelay networks.

To derive closed-form expressions for the achievable rate of the proposed scheme, we restrict ourselves to the superposition broadcast encoding. In addition, we also restrict ourselves to a linear ordering of relays and a loop-free relationship between the messages and the parities. Thus, the rates derived in this paper do not utilize the possibility of parallel relaying [17], or the ability for multiple nodes in a network with cycles to discuss back and forth with each other. Further generalization of this work is possible by using more advanced broadcast schemes and more flexible relay topologies.

\section{REFERENCES}

[1] E. C. van der Meulen, "Three-terminal communication channels," $A d v$. Appl. Prob., vol. 3, pp. 120-154, 1971.

[2] L. L. Xie and P. R. Kumar, "An achievable rate for the multiple-level relay channel," IEEE Trans. Inf. Theory, vol. 51, no. 4, pp. 1348-1358, Apr. 2005.

[3] P. Gupta and P. R. Kumar, "Towards an information theory of large networks: An achievable rate region," IEEE Trans. Inf. Theory, vol. 49, no. 8, pp. 1877-1894, Aug. 2003.

[4] G. Kramer, M. Gastpar, and P. Gupta, "Cooperative strategies and capacity theorems for relay networks," IEEE Trans. Inf. Theory, vol. 51, no. 9, pp. 3037-3063, Sept. 2005.

[5] A. Reznik, S. R. Kulkarni, and S. Verdú, "Degraded Gaussian multirelay channel: Capacity and optimal power aladdress," IEEE Trans. Inf. Theory, vol. 50, no. 12, pp. 3037-3046, Dec. 2004.

[6] M. Gastpar and M. Vetterli, "On the capacity of large Gaussian relay networks," IEEE Trans. Inf. Theory, vol. 51, no. 3, pp. 765-779, Mar. 2005.

[7] P. Rost and G. Fettweis, "A generalized mixed strategy for multiterminal relay networks," in Proc. IEEE Inf. Theory Workshop (ITW), Lake Tahoe, California, Sept. 2007, pp. 366-371.

[8] T. M. Cover and A. A. El Gamal, "Capacity theorems for the relay channel," IEEE Trans. Inf. Theory, vol. 25, no. 5, pp. 572-584, Sept. 1979.

[9] M. R. Aref, "Information Flow in Relay Networks," Ph.D. dissertation, Stanford University, Stanford, CA, Oct. 1980.

[10] A. El Gamal and M. R. Aref, "The capacity of the semideterministic relay channel," IEEE Trans. Inf. Theory, vol. 28, no. 3, pp. 536-536, May 1982.

[11] L. Ghabeli and M. R. Aref, "A new achievable rate and the capacity of some classes of multilevel relay network," EURASIP J. Wireless Commun. Netw., vol. 2008, 2008, Article ID: 135857.

[12] A. S. Avestimehr, S. N. Diggavi, and D. N. C. Tse, "A deterministic model for wreless relay networks an its capacity," in Proc.IEEE Inf. Theory Workshop (ITW), Lake Tahoe, CA, Sep. 2007, pp. 1-6.
[13] A. S. Avestimehr, S. N. Diggavi, and D. N. C. Tse, "Approximate capacity of gaussian relay networks," in Proc. of IEEE Int. Symp. Inform. Theory (ISIT), Toronto, ON, Canada, Jul. 2008, pp. 474-478.

[14] A. Wyner, "Recent results in the Shannon theory," IEEE Trans. Inf Theory, vol. 20, no. 1, pp. 2-10, Jan. 1974.

[15] P. Razaghi and W. Yu, "Bilayer low-density parity-check codes for decode-and-forward in relay channels," IEEE Trans. Inf. Theory, vol. 53, no. 10, pp. 3723-3739, Oct. 2007.

[16] P. Gupta and P. R. Kumar, "The capacity of wireless networks," IEEE Trans. Inf. Theory, vol. 46, pp. 388-404, Mar. 2000.

[17] B. Schein and R. Gallager, "The Gaussian parallel relay network," in Proc. IEEE Int. Symp. Inf. Theory (ISIT), Sorrento, Italy, June 2000, p. 22.

[18] M. Gastpar, G. Kramer, and P. Gupta, "The multiple-relay channel Coding and antenna-clustering capacity," in Proc. IEEE Int. Symp. Inf. Theory (ISIT), Laussane, Switzerland, Jul. 2002, p. 136.

[19] L.-L. Xie and P. R. Kumar, "A network information theory for wireless communication: Scaling laws and optimal operation," IEEE Trans. Inf. Theory, vol. 50, no. 5, pp. 748-767, May 2004.

[20] A. B. Carleial, "Multiple-access channels with different generalized feedback signals," IEEE Trans. Inf. Theory, vol. 28, no. 6, pp. 841-850, Nov. 1982.

[21] T. M. Cover and J. A. Thomas, Elements of Information Theory, 2nd ed. New York: Wiley, 2006.

[22] A. El Gamal and S. Zahedi, "Capacity of a class of relay channels with orthogonal components," IEEE Trans. Inf. Theory, vol. 51, no. 5, pp. 1815-1817, May 2005.

Peyman Razaghi (S'99) was born in Isfahan, Iran, 1980. He received the B.Sc degree in electrical engineering and the M.Sc. degree in communication systems from Sharif University of Technology, Tehran, Iran, in 2002 and 2004, respectively. He is currently pursuing the Ph.D. degree with the Department of Electrical and Computer Engineering, University of Toronto, Canada.

His research interests are in the areas of multiuser information theory, wireless communications, coding, and signal processing. During his Master and $\mathrm{Ph} . \mathrm{D}$. studies, he has been working on complexity manageable space-time codes, coding for the relay channel, relaying protocols for multirelay networks and characterization of the capacity of relay channels.

Wei Yu (S'02-M'02-SM'08) received the B.A.Sc. degree in computer engineering and mathematics from the University of Waterloo, Waterloo, ON, Canada, in 1997 and the M.S. and Ph.D. degrees in electrical engineering from Stanford University, Stanford, CA, in 1998 and 2002, respectively.

Since 2002, he has been Edward Rogers Sr. with the Department of Electrical and Computer Engineering at the University of Toronto, Toronto, ON, Canada, where he is now an Associate Professor and holds a Canada Research Chair in Information Theory and Digital Communiations. His main research interests include multiuser information theory, optimization, wireless communications, and broadband access networks.

Prof. $\mathrm{Yu}$ was an Editor for IEEE TRANSACTIONS ON WIRELESS COMMUNicATIONS from 2004 to 2007. He was a Guest Editor of IEEE JOURNAL ON SELECTED AREAS IN COMMUNICATIONS for a special issue on "Nonlinear Optimization of Communications Systems" in 2006, and a Guest Editor of EURASIP Journal on Applied Signal Processing for a special issue on "Advanced Signal Processing for Digital Subscriber Lines" in 2005. He received Early Researcher Award from Ontario in 2006, and the Early Career Teaching Award from the Faculty of Applied Science and Engineering, University of Toronto, in 2007. 